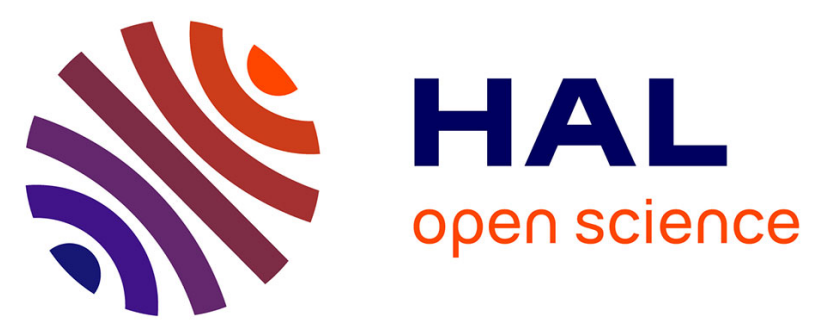

\title{
Large Eddy Simulation of Swirled Spray Flame Using Detailed and Tabulated Chemical Descriptions
}

\author{
B. Franzelli, A. Vié, M. Boileau, B. Fiorina, N. Darabiha
}

\section{To cite this version:}

B. Franzelli, A. Vié, M. Boileau, B. Fiorina, N. Darabiha. Large Eddy Simulation of Swirled Spray Flame Using Detailed and Tabulated Chemical Descriptions. Flow, Turbulence and Combustion, 2017, 98 (2), pp.633 - 661. 10.1007/s10494-016-9763-0 . hal-01480274

\section{HAL Id: hal-01480274 https://hal.science/hal-01480274}

Submitted on 13 Mar 2017

HAL is a multi-disciplinary open access archive for the deposit and dissemination of scientific research documents, whether they are published or not. The documents may come from teaching and research institutions in France or abroad, or from public or private research centers.
L'archive ouverte pluridisciplinaire HAL, est destinée au dépôt et à la diffusion de documents scientifiques de niveau recherche, publiés ou non, émanant des établissements d'enseignement et de recherche français ou étrangers, des laboratoires publics ou privés. 


\title{
Large Eddy Simulation of swirled spray flame using detailed and tabulated chemical descriptions
}

\author{
B. Franzelli, A. Vié, M. Boileau, B. Fiorina, N. \\ Darabiha
}

the date of receipt and acceptance should be inserted later

\begin{abstract}
Accurate characterization of swirled flames is a key point in the development of more efficient and safer aeronautical engines. The task is even more challenging for spray injection systems. From one side, spray interacts with both turbulence and flame, eventually affecting the flame dynamics. On the other side, the structure of turbulent spray flame is highly complex due to equivalence ratio inhomogeneities caused by evaporation and mixing processes. The first objective of this work is to numerically characterize the structure and dynamics of a swirled spray flame. The target configuration is the experimental benchmark named MERCATO, representative of an actual turbojet injection system. Due to the complex nature of the flame, a detailed description of chemical kinetics is necessary and is here obtained by using a 24-species chemical scheme, which has been expressly developed for DNS of spray flames. The first LES of a swirled spray flame using such a detailed chemical description is performed here and results are analyzed to study the complex interactions between the spray, the turbulent flow and the flame. It is observed that this coupling has an effect on the flame structure and that flame dynamics are governed by the interactions between spray, precessing vortex core and flame front. Even if such a detailed kinetic description leads to an accurate characterization of the flame, it is still highly expensive in terms of CPU time. Tabulated techniques have been expressly developed to account for detailed chemistry at a reduced computational cost in purely gaseous configurations. The second objective is then to verify the capability of the FPI tabulated chemistry method to correctly reproduce the spray flame characteristics by performing LES. To do this, results with the FPI method are compared to the experimental database and to the results obtained with the 24-species description in terms of mean and fluctuating axial gas velocity and liquid phase characteristics (droplet diameter and liquid velocity). Moreover, the flame characterization obtained with the FPI approach is compared to the results of the 24-species scheme focusing on the flame structure, on major and minor species concentrations as well as on pollutant emissions. The potential and the limits of the tabulated approach for spray flame are finally assessed.
\end{abstract}

Laboratoire EM2C, CNRS, CentraleSuplec, Universit Paris-Saclay,

Grande Voie des Vignes, 92295 Châtenay-Malabry Cedex, FRANCE

Email: benedetta.franzelli@cnrs.fr, aymeric.vie@centralesupelec.fr, matthieu.boileau@math.unistra.fr, benoit.fiorina@centralesupelec.fr, nasser.darabiha@centralesupelec.fr 


\section{Introduction}

Numerical tools for spray combustion help engineers to design more efficient and less pollutant aeronautical engines. In terms of modeling, the task remains extremely challenging as spray flames present a complex nature, comprising atomization, evaporation, mixing and combustion. As fuel droplet evaporation causes strong inhomogeneities of equivalence ratio in fresh gases, the flame structure and dynamics are highly complex. In this stratified and multi-regime environment, different combustion modes (i.e. premixed, partially-premixed and non-premixed) may be simultaneously observed in spray flames [1]. A realistic chemical description is crucial if the combustion phenomena that have to be reproduced are sensitive to detailed chemistry, such as flame stabilization and pollutant emissions.

The direct use of detailed chemical description in practical simulations is necessary for the prediction of flame stabilization, ignition and pollutant concentration, but is also expensive in terms of CPU time, since the computational time depends on the number of transported species. Tabulated chemistry methods, based for example on premixed flames [2,3], have then been proposed to overcome this issue. As shown in [4], using tabulated chemistry is an efficient way to save CPU time since few transport equations for the table coordinates are solved instead of the species transport equations, without loss of accuracy on laminar premixed flames. Tabulated chemistry methods have been widely used to introduce detailed chemistry in Large Eddy Simulation (LES) of turbulent gaseous flames [5-7] at a reduced computational cost. These techniques seem also attractive for spray combustion and they have already been used to perform DNS (Direct Numerical Simulation) and LES of turbulent spray flames $[1,8-10]$. However, their capabilities to correctly reproduce spray flame structure and pollutants still have to be evaluated rigorously.

In this context, the objectives of this work are twofold. First, a numerical characterization of a swirled spray flame stabilized in a practical turbojet injection system is provided using a 24-species chemical scheme [11]. For that purpose, the MERCATO (Moyen Exprimental de Recherche en Combustion Arobie par Techniques Optiques) ${ }^{1}$ experimental benchmark is retained. This configuration is representative of the two-phase flow produced by an industrial swirl air/kerosene turbojet injection system. It has been widely described and studied experimentally [12]. Moreover, numerical studies are available for the evaporation phenomena under non-reactive conditions [13-15]. The use of a detailed chemical description in LES of the spray flow allows the investigation of the complex interactions between the spray, the turbulent flow and the flame front and their effects on the flame structure.

The second objective is to investigate the capability of the standard FPI (Flame Prolongation of ILDM) tabulated chemistry method [2] to reproduce swirled spray flames by performing LES of the MERCATO configuration. Results are also compared to those of the 24-species chemistry to assess the accuracy of the tabulated method.

This article is organized as follows. Multi-species and tabulated chemical descriptions are introduced in Section 2. Then the Large Eddy Simulation methodology for spray flow is presented. The system of equations for both gas and spray flows using the Euler-Euler approach is first detailed for the multi-species chemistry and then extended to the FPI method in Section 3. The experimental configuration as well as the numerical setup are presented in Section 4. The validation of the retained numerical approach is presented in Section 4.3, by

\footnotetext{
1 Experimental setup for investigation of air-breathing combustion using optical techniques.
} 
comparing results on axial velocity of both phases as well as the mean droplet diameter to the experimental data. Then, the turbulent reactive two-phase flow is characterized in Section 5. Based on the reference multi-species calculation, the flame structure is analyzed in Section 5.1 while the spray-precessing vortex core-flame interactions, which have not been analyzed experimentally, are investigated in Section 5.2. Finally, the quality of the FPI lookup table approach is assessed in Section 6 by comparing the results with the data from the multi-species calculation.

\section{Chemical description}

In the following, the two different chemical descriptions considered, i.e. a multi-species kinetics and the FPI look-up table technique, are presented. Kerosene is modeled here by n-dodecane $\left(\mathrm{C}_{12} \mathrm{H}_{26}\right)$.

\subsection{Multi-species chemistry}

A 24-species mechanism developed to perform DNS of n-dodecane spray flames $[11,16]$ is considered here. It consists of an analytical reconstruction of the species chemical production rates $\dot{\omega}_{k}$ from the detailed JetSurF 1.0 mechanism [17], originally consisting of 123 species and 977 reactions. The reduction is obtained using directed relation graph (DRG), DRG aided sensitivity analysis and linearized quasi steady state (QSS) approximations in auto-ignition and perfectly-stirred reactors. It guarantees a correct description of the laminar flame structure and its response to strain rate variations. By preserving the main chemical pathways, the 24-species chemistry provides an accurate detailed description of the chemical processes for spray flames, which is not guaranteed when using 'ad hoc' fitted multi-species chemistries [18-20] or tabulated techniques $[1,21]$ for simulations of turbulent spray flames $[8,10,22-27]$.

\subsection{Tabulated chemistry}

The tabulated chemistry method retained in this work is the FPI method [2], which assumes that the chemical subspace accessed by a flame can be mapped by a collection of 1-D gaseous laminar premixed flames computed using detailed chemistry for various equivalence ratios $\phi_{L}<\phi<\phi_{R}$ within the flammability limits $\phi_{L}$ and $\phi_{R}$. In this method, the composition space is determined by a reduced set of variables such as the progress variable

$$
Y_{\mathrm{C}}=Y_{\mathrm{CO}}+Y_{\mathrm{CO}_{2}}+Y_{\mathrm{H}_{2} \mathrm{O}}
$$

which evolves monotonically between fresh and burnt gases. The mixture fraction, defined as

$$
Y_{z}=\frac{W_{F}}{W_{C} n_{C F}} \sum_{k=1}^{N_{s p e c}} Y_{k} \frac{n_{C k} W_{C}}{W_{k}}
$$

is often retained as a parameter of a look-up table method, where $W_{C}$ is the element weight of carbon atom, $Y_{k}, W_{k}$ and $n_{C k}$ are the mass fraction, the molar weight and the number of carbon atoms of the $\mathrm{k}^{\text {th }}$ species, respectively. Subscript $F$ stands for the fuel index. Under the unity Lewis number assumption, the mixture fraction represents the local mixture 
equivalence ratio. Any thermo-chemical quantity $\varphi$ is then stored in a 2-D look-up table $\varphi=\varphi^{\mathrm{FPI}}\left[Y_{c}, Y_{z}\right]$, where $\varphi^{\mathrm{FPI}}$ is obtained from laminar premixed flames. This chemical tabulation procedure does not reproduce the heat exchange from the liquid to the gas phase since the gas temperature $T$ is directly read from an adiabatic chemical table $T=T^{\mathrm{FPI}}$.

One possibility to take into account heat exchanges is to build the table with non-adiabatic flamelets [28,29]. An additional coordinate, the gas mixture enthalpy $h$, is then used to build the look-up table. However, adding a third dimension to the table increases memory and CPU time.

In this work a simpler approach has been retained based on a $Y_{c}-Y_{z}$ look-up table built from purely gaseous adiabatic freely propagating premixed flames by assuming that the chemical composition is slightly sensitive to small energy fluctuations so that heat exchanges between phases mainly affect the temperature. To take into account this heat transfer, the Tabulated Thermochemistry for Compressible flows formalism (TTC) [4] is considered here, so that the gas temperature $T$ is a first-order linearization of the transported energy $E$ using the tabulated energy $E^{\mathrm{FPI}}$ and the tabulated gas temperature $T^{\mathrm{FPI}}$ :

$$
T=T^{\mathrm{FPI}}\left(Y_{c}, Y_{z}\right)+\frac{E-E^{\mathrm{FPI}}\left(Y_{c}, Y_{z}\right)}{c_{v}^{\mathrm{FPI}}\left(Y_{c}, Y_{z}\right)}
$$

where $c_{v}^{\mathrm{FPI}}$ is the tabulated mixture heat capacity at constant volume. In such a way, the flow temperature takes into account the heat exchange due to the droplet evaporation as well the energy fluctuations due to compressible phenomena.

Performances of the FPI tabulated approach on laminar counterflow spray flames have been investigated in 'a priori' way in [21]. An 'a posteriori' analysis is proposed in Appendix A. Even if the prediction of intermediate species requires more sophisticated models $[21,30]$, the global behavior of laminar spray flames is correctly reproduced by the FPI method. It is however not straightforward to anticipate the consequences of the discrepancies observed on laminar spray flames in the case of the LES prediction of a swirled turbulent spray flame. The tabulated approach is expected to successfully reproduce a turbulent flame if its structure belongs to the tabulated manifold, but this is not ensured in the case of swirled spray flames due to the their complex structure. For this reason, the adequacy of the FPI technique for LES of turbulent swirled spray flames is assessed in Section 6 by comparing directly its performance in a LES simulation of the MERCATO burner with the results obtained with the 24-species description.

\section{LES system of equations}

LES of the MERCATO spray flame configuration is performed with the AVBP solver [3133] using an Euler-Euler approach under the assumption of monodisperse-monokinetic liquid phase. This assumption may affect the accuracy of the spray description but significantly reduces the simulation CPU cost. The effect of such assumptions on the results will be discussed in Section 4.3. 
3.1 Gas phase description

According to $[13,14]$, the filtred conservation equations for mass $\rho$, momentum $\rho \mathbf{u}$, energy $E$ and species mass fraction $Y_{k}$ in a two-phase flow for a very dilute regime ${ }^{2}$ are given by:

$$
\begin{gathered}
\frac{\partial \bar{\rho}}{\partial t}+\frac{\partial \bar{\rho} \tilde{u}_{j}}{\partial x_{j}}=\frac{\bar{\Gamma}}{\mathcal{F}} \\
\frac{\partial \bar{\rho} \tilde{u}_{i}}{\partial t}+\frac{\partial \bar{\rho} \tilde{u}_{i} \tilde{u}_{j}}{\partial x_{j}}=-\frac{\partial}{\partial x_{j}}\left[\bar{p} \delta_{i j}-\bar{\tau}_{i j}-\bar{\tau}_{i j}^{s g s}\right]+\tilde{u}_{l, i} \frac{\bar{\Gamma}}{\mathcal{F}}-\bar{F}_{d, i} \\
\frac{\partial \bar{\rho} \tilde{E}}{\partial t}+\frac{\partial \bar{\rho} \tilde{E} \tilde{u}_{j}}{\partial x_{j}}=-\frac{\partial}{\partial x_{j}}\left[\overline{u_{i}\left(p \delta_{i j}-\tau_{i j}\right)}+\mathcal{E} \mathcal{F} \bar{q}_{j}+\bar{q}_{j}^{s g s}\right] \\
+\frac{\bar{\Pi}}{\mathcal{F}}+\left(\frac{1}{2} \tilde{u}_{l, i}^{2}\right) \frac{\bar{\Gamma}}{\mathcal{F}}-\tilde{u}_{l, i} \bar{F}_{d, i} \\
\frac{\partial \bar{\rho} \tilde{Y}_{k}}{\partial t}+\frac{\partial \bar{\rho} \tilde{Y}_{k} \tilde{u}_{j}}{\partial x_{j}}=\frac{\partial}{\partial x_{j}}\left[\mathcal{E \mathcal { F }} \bar{J}_{k, j}+\bar{J}_{k, j}^{s g s}\right]+\frac{\mathcal{E} \overline{\dot{\omega}}_{k}}{\mathcal{F}}+\frac{\bar{\Gamma} \delta_{k F}}{\mathcal{F}} k=1, N_{s}
\end{gathered}
$$

where $\cdot$ and $\sim$ represent the Reynolds and the Favre spatial filtering respectively, $\delta_{i j}$ corresponds to the Kronecker symbol. $p$ is the pressure and $\dot{\omega}_{k}$ is the mass production rate of $k^{\text {th }}$ species. $\mathbf{F}_{\mathbf{d}}$ is the drag-force evaluated using the Schiller-Nauman correlation [34]. $\Gamma$ and $\Pi$ are the mass and energy source terms due to evaporation. $\delta_{k F}$ is equal to 1 when $k$ corresponds to the fuel and 0 for all other species. $\mathcal{F}$ and $\mathcal{E}$ are the thickening and efficiency factors required by the dynamically thickened flame subgrid model detailed below. The filtered laminar stress tensor $\tau_{i j}$, the heat flux $q_{j}$ and the diffusive species flux $J_{k, j}$ follow the classical formulations [35]:

$$
\begin{aligned}
& \bar{\tau}_{i j}=2 \bar{\mu} \tilde{S}_{i j}, \quad \bar{q}_{j}=\bar{\lambda} \frac{\partial \tilde{T}}{\partial x_{i}}+\sum_{k=1}^{N_{s}} \bar{J}_{i, k} \tilde{h}_{s, k} \\
& \bar{J}_{k, j}=\bar{\rho} \bar{D}_{k} \frac{\partial \tilde{X}_{k}}{\partial x_{j}}-\bar{\rho}_{k} \bar{V}_{k, j}, \quad \bar{V}_{k, j}=\sum_{k=1}^{N_{s}} \bar{D}_{k} \frac{W_{k}}{\bar{W}} \frac{\partial \tilde{X}_{k}}{\partial x_{i}},
\end{aligned}
$$

where $\tilde{S}_{i j}$ is the shear tensor of the resolved field, $\tilde{h}_{s, k}$ is the filtered sensible enthalpy of the species $k, \bar{X}_{k}$ is the filtered molar fraction of the $\mathrm{k}^{t h}$ species, and $\bar{V}_{k, j}$ is the correction velocity to ensure mass conservation. For the subgrid unclosed term $\tau_{i j}^{s g s}$, a viscosity-type closure is used:

$$
\bar{\tau}_{i j}^{s g s}=2 \mu^{t} \tilde{S}_{i j}-\frac{1}{3} \bar{\tau}_{k k}^{s g s} \delta_{i j}
$$

and the turbulent viscosity $\mu^{t}$ is evaluated using the WALE model of Nicoud et al. [36]. For subgrid heat flux $q_{j}^{s g s}$ and species diffusion flux $J_{i, k}^{s g s}$, constant turbulent Prandtl and Schmidt numbers $\operatorname{Pr}_{t}=\mu^{t} \bar{c}_{p} / \lambda^{t}$ and $\mathrm{Sc}^{t}=\mu^{t} / \bar{\rho} D_{k}^{t}$ are assumed along with the following closures:

$\bar{q}_{j}^{t}=\lambda^{t} \frac{\partial \tilde{T}}{\partial x_{i}}+\sum_{k=1}^{N} \bar{J}_{i, k}^{t} \tilde{h}_{s, k}, \quad \bar{J}_{k, j}^{t}=\bar{\rho} D_{k}^{t} \frac{\partial \tilde{X}_{k}}{\partial x_{j}}-\bar{\rho} \tilde{Y}_{k} \bar{V}_{k, j}^{t}, \quad \bar{V}_{k, j}^{t}=\sum_{k=1}^{N_{s}} \bar{D}_{k}^{t} \frac{W_{k}}{\bar{W}} \frac{\partial \tilde{X}_{k}}{\partial x_{i}}$.

\footnotetext{
${ }^{2}$ In the very dilute regime, the volume occupation of the liquid phase is negligible.
} 
The thickening factor $\mathcal{F}$ and the efficiency function $\mathcal{E}$ in Eqs. (4-7) are linked to the DynamicallyThickened Flame (DTFLES) model [33], which allows the correct numerical representation of the flame on a LES mesh for which the mesh resolution is not sufficient to reproduce the flame thickness. The thickening factor $\mathcal{F}$ increases the molecular diffusion and decreases the reaction rate to thicken the flame front while preserving the laminar flame speed. The efficiency function $\mathcal{E}$ accounts for the fact that the thickening is lowering the flame wrinkling [37]. Here, a sensor is used to locally activate the thickening, based on the chemical reaction rate of the progress variable $\dot{\omega}_{Y_{c}}$ [38], which localizes the inner reaction zone and the postlame zone ${ }^{3}$. Once activated, the thickening factor $\mathcal{F}$ guarantees that the flame front is numerically discretized at least by ten grid points. In the thickened region, turbulent fluxes are not applied as these effects are already taken into account in the thickened flame model. The evaporation term $\Gamma$ is also divided by $\mathcal{F}$ in Eqs. (4-7). Even if the droplet evaporation is then altered close to the flame front, such strategy preserves the Lefebvre number of the flame, i.e. the ratio between the evaporation time and the chemical time, which is a key parameter for the characterization of spray flames[16].

\subsubsection{Extension to the tabulated description}

In the FPI formulation, species balance equations (Eq. (7)) are replaced by one equation for the mixture fraction $Y_{z}$ and one for the progress variable $Y_{c}$ :

$$
\begin{aligned}
& \frac{\partial \bar{\rho} \tilde{Y}_{z}}{\partial t}+\frac{\partial \bar{\rho} \tilde{Y}_{z} \tilde{u}_{j}}{\partial x_{j}}=\frac{\partial}{\partial x_{j}}\left[\mathcal{E} \mathcal{F} \bar{J}_{j, Y_{z}}+\bar{J}_{j, Y_{z}}^{s g s}\right]+\frac{\bar{\Gamma}}{\mathcal{F}} \\
& \frac{\partial \bar{\rho} \tilde{Y}_{c}}{\partial t}+\frac{\partial \bar{\rho} \tilde{Y}_{c} \tilde{u}_{j}}{\partial x_{j}}=\frac{\partial}{\partial x_{j}}\left[\mathcal{E} \mathcal{F} \bar{J}_{j, Y_{c}}+\bar{J}_{j, Y_{c}}^{s g s}\right]+\frac{\mathcal{E} \overline{\dot{\omega}}_{Y_{c}}^{\mathrm{FPI}}}{\mathcal{F}} .
\end{aligned}
$$

The mass transfer $\Gamma$ from liquid to gas due to the evaporation is accounted for in the balance equation of the gaseous mixture fraction $Y_{z}$. The diffusive flux of the progress variable $J_{i, Y_{c}}$ and that of the mixture fraction $J_{i, Y_{z}}$ as well as the corresponding unclosed subgrid terms are closed using Eq. (9) and Eq. (11), respectively.

\subsubsection{Thermodynamic and transport properties of the gaseous mixture}

In the multi-species description, all thermodynamic quantities are derived from enthalpy and entropy information for each species based on the JANAF tables [39]. Concerning the transport properties, a simplified model based on constant and equal Schmidt (Sc) and Prandtl (Pr) numbers, ${ }^{4}$ i.e. unity Lewis number for all species, is considered here in order to guarantee consistency with the tabulated chemistry. The gas diffusivity of the $\mathrm{k}^{\text {th }}$ species is then $D_{k}=D=\frac{\mu \mathrm{Sc}}{\rho}$ where the dynamic viscosity $\mu$ is obtained via a classical power law $\mu=\mu_{0}\left(T / T_{0}\right)^{\alpha_{P}}$, with $T_{0}=473 \mathrm{~K}, \mu_{0}=2.5034 e^{-5}[\mathrm{~kg} / \mathrm{m} / \mathrm{s}]$ and $\alpha_{P}=0.6695$ [20]. The heat conductivity $\lambda$ is obtained as: $\lambda=\frac{\mu c_{p}}{\operatorname{Pr}}$ where $c_{p}$ is the heat capacity at constant pressure calculated at temperature $T$ :

$$
c_{p}=\sum_{k=1}^{N_{s}} c_{p}^{k}(T) Y_{k}
$$

\footnotetext{
3 To ensure consistency between the two chemical descriptions, the same definition of the sensor is used in both calculations. In the case of the detailed chemical description, the reaction rate of the progress variable is reconstructed from the transported species.

4 Here the values for Schmidt and Prandtl numbers are $\mathrm{Sc}=0.7$ and $\mathrm{Pr}=0.7$, respectively [20].
} 
where $c_{p}^{k}$ is reconstructed from enthalpy tables.

In the tabulated approach, any transport or thermodynamic property $\varphi$ of the gaseous mixture is calculated from adiabatic freely propagating premixed laminar flames imposing unity Lewis number for all species and stored in the FPI look-up table as a function of $Y_{c}$ and $Y_{z}: \varphi=\varphi^{\mathrm{FPI}}\left(Y_{c}, Y z\right)$. As a consequence, the extracted values for $D_{k}^{\mathrm{FPI}}$ and $\lambda^{\mathrm{FPI}}$ correspond to specific species composition $Y_{k}^{\mathrm{FPI}}\left(Y_{c}, Y z\right)$ and temperature $T^{\mathrm{FPI}}\left(Y_{c}, Y z\right)$. It has to be noted that the flow temperature $T$ obtained from Eq. (3) may differ from the tabulated value $T^{\mathrm{FPI}}$. Such discrepancy may lead to some inaccuracies on the thermodynamic and transport properties. However, it has been verified in the LES calculations that the difference $\Delta T=T-T^{\mathrm{FPI}}$ between the flow temperature $T$ and the tabulated value is generally lower than $50 \mathrm{~K}$ and confined in a small region close to the injection. The impact of these discrepancies on thermodynamic and transport properties is then not significant.

The use of such simplified properties in both multi-species and tabulated simulations, together with the other assumptions due to the LES modelling, may affect the results and may lead to some discrepancies with the experimental observations. However, they allow a straightforward comparison with the results from the tabulated technics, since both LES simulations will rely on the same assumptions.

\subsection{Spray flow filtred balance equations}

In the Euler-Euler approach the disperse phase, considered as a continuum, is described using macroscopic quantities, here the droplet number density $n_{l}$, the liquid velocity $\mathbf{u}_{l}$, the liquid enthalpy $h_{l}$ and the liquid volume fraction $\alpha_{l}=\pi n_{l} d_{l}^{3} / 6$ ( $d_{l}$ is the droplet diameter). Considering monodisperse, monokinetic ${ }^{5}$ mono-component fuel droplets in a very dilute regime, the filtered conservation equations for the liquid phase are the following [40]:

$$
\begin{aligned}
\frac{\partial \bar{n}_{l}}{\partial t}+\frac{\partial \bar{n}_{l} \tilde{u}_{l, j}}{\partial x_{j}} & =0 \\
\frac{\partial \rho_{l} \bar{\alpha}_{l}}{\partial t}+\frac{\partial \rho_{l} \bar{\alpha}_{l} \tilde{u}_{l, j}}{\partial x_{j}} & =-\frac{\bar{\Gamma}}{\mathcal{F}} \\
\frac{\partial \rho_{l} \bar{\alpha}_{l} \tilde{u}_{l, i}}{\partial t}+\frac{\partial \rho_{l} \bar{\alpha}_{l} \tilde{u}_{l, i} \tilde{u}_{l, j}}{\partial x_{j}} & =\frac{\partial \bar{\tau}_{l, i j}^{s g s}}{\partial x_{j}}+\rho_{l} \bar{\alpha}_{l} \bar{F}_{d, i}-\tilde{u}_{l, i} \frac{\bar{\Gamma}}{\mathcal{F}} \\
\frac{\partial \rho_{l} \bar{\alpha}_{l} \tilde{h}_{l}}{\partial t}+\frac{\partial \rho_{l} \bar{\alpha}_{l} \tilde{u}_{l, j} \tilde{h}_{l}}{\partial x_{j}} & =-\frac{\bar{\Pi}}{\mathcal{F}}
\end{aligned}
$$

where $\rho_{l}=750 \mathrm{~kg} \cdot \mathrm{m}^{-3}$ is the liquid density of dodecane and $\bar{\tau}_{l}^{\text {sgs }}$ is the particle subgrid stress tensor closed using the Smagorinsky-Yoshizawa model [40]. The SmagorinskyYoshizawa model is similar to the Smagorinsky model for gas flows, but in addition it takes into account a subgrid pressure. While being negligible in incompressible flow, this subgrid pressure may become predominant in highly compressible flows, such as the Eulerian disperse phase. The filtered source terms $\bar{\Gamma}$ and $\bar{\Pi}$ and all the modeling closures on the disperse

\footnotetext{
5 Monodisperse and monokinetic assumptions imply that at a given location all droplets have the same size and velocity, i.e. Dirac's delta distributions in size and velocity phase spaces.
} 
phase are the same as in [14]:

$$
\begin{aligned}
& \bar{\Gamma}=\pi \bar{n}_{l} d_{l} \operatorname{Sh} \rho D^{\text {evap }} \ln (1+B) \\
& \bar{\Pi}=\pi \bar{n}_{l} d_{l} \operatorname{Nu} \rho \lambda^{\text {evap }}\left(\tilde{T}-\tilde{T}_{l}\right) \frac{\ln (1+B)}{B}-\bar{\Gamma}\left(L_{v}\left(\tilde{T}_{l}\right)-\tilde{h}_{l}\right)
\end{aligned}
$$

where $\mathrm{Nu}$ and $\mathrm{Sh}$ are the Nusselt and Sherwood numbers, respectively. $B$ is the Spalding number, $D^{\text {evap }}$ and $\lambda^{\text {evap }}$ are the reference gas diffusivity of fuel and conductivity close to the droplet surface, respectively, and $L_{v}$ is the latent heat of liquid. One of the key points in the evaluation of the vaporization rate is the reference gas diffusivity which is affected by the composition and the temperature [41]. The retained formulation for the thermodynamic and transport properties at the droplet surface in the case of a multi-species chemistry is provided in the following section together with its extension to the tabulated approach.

\subsubsection{Transport and thermodynamic properties in the droplet vicinity}

In analogy with the gaseous mixture treatment discussed in Section 3.1.1, $D^{\text {evap }}$ and $\lambda^{\text {evap }}$ are calculated with both multi-species and tabulated approaches by using constant Schmidt and Prandtl numbers assumptions: $\rho D^{\text {evap }}=\mu^{\text {evap }} \mathrm{Sc}^{-1}$ and $\lambda^{\text {evap }}=\mu^{\text {evap }} c_{p}^{\text {evap }} \operatorname{Pr}^{-1}$. The reference viscosity $\mu^{\text {evap }}$ and conductivity $\lambda^{\text {evap }}$ are calculated using the so-called " $2 / 3-1 / 3$ " approximation from the reference temperature $T^{\text {evap }}$ and composition $Y_{k}^{\text {evap }}$ close to the droplet surface[42]:

$$
\mu^{\text {evap }}=\mu\left(Y_{k}^{\text {evap }}, T^{\text {evap }}\right), \quad c_{p}^{\text {evap }}=c_{p}\left(Y_{k}^{\text {evap }}, T^{\text {evap }}\right) .
$$

with:

$$
T^{\text {evap }}=\frac{2}{3} T_{l}+\frac{1}{3} T_{g}, \quad Y_{F}^{\text {evap }}=\frac{2}{3} Y_{F}^{\text {surf }}+\frac{1}{3} Y_{F}, \quad Y_{k}^{\text {evap }}=\frac{Y_{k}\left(1-Y_{F}^{\text {evap }}\right)}{1-Y_{F}}
$$

where $Y_{F}^{\text {surf }}$ is the fuel mass fraction at the droplet surface [41].

With the multi-species description, the reference heat capacity is then calculated:

$$
c_{p}^{\text {evap }}=\sum_{k=1}^{N_{s}} c_{p}^{k}\left(T^{\text {evap }}\right) Y_{k}^{\text {evap }} .
$$

Following Sierra et al. [41], the molecular viscosity is not anymore assumed to be independent from the gas composition and is obtained with the Wilke formula [43]:

$$
\mu^{\text {evap }}=\sum_{k=1}^{N_{s}} \frac{X_{k}^{\text {evap }} \mu_{k}^{\text {evap }}}{\sum_{l=1}^{N_{s}} X_{l}^{\text {evap }} \phi_{k l}^{\text {evap }}},
$$

where $X_{k}$ is the molar fraction of species $k, N_{s}$ is the number of species accounted for in the chemical model and

$$
\phi_{k l}^{\text {evap }}=\frac{1}{\sqrt{8}}\left(1+\frac{W_{k}}{W_{l}}\right)^{-1 / 2}\left[1+\left(\frac{\mu_{k}^{\text {evap }}}{\mu_{l}^{\text {evap }}}\right)^{1 / 2}\left(\frac{W_{l}}{W_{k}}\right)^{1 / 4}\right] .
$$

The dynamic viscosity of the $\mathrm{k}^{\text {th }}$ species $\mu_{k}^{\text {evap }}$ follows a power-law:

$$
\mu_{k}^{\text {evap }}=\mu_{k, 0}\left(\frac{T^{\text {evap }}}{T_{0}}\right)^{\alpha_{k}}
$$


where $\mu_{k, 0}$ is the viscosity of the $\mathrm{k}^{t h}$ species at the temperature $T_{0}$ and $\alpha_{k}$ is the power-law coefficient allowing a correct description of the viscosity on a wide range of temperature.

In contrast with the gaseous mixture description, a special treatment is necessary when using the tabulated approach for the calculation of $c_{p}^{\text {evap }}$ and $\mu^{\text {evap }}$. In fact, the stored values may be inaccurate when $T^{\mathrm{evap}}$ is far from $T^{\mathrm{FPI}}$. This situation may be encountered for example when evaporation occurs in high-temperature region, i.e. when liquid and gaseous phases are far from thermal equilibrium $T_{l}=T_{g}$. To reconstruct the reference properties close to the droplet surface, the mass fraction $Y_{k}=Y_{k}^{F P I}$ of the six major species $\left(\mathrm{C}_{12} \mathrm{H}_{26}, \mathrm{O}_{2}\right.$, $\mathrm{N}_{2}, \mathrm{CO}_{2}, \mathrm{CO}, \mathrm{H}_{2} \mathrm{O}$ ) are then extracted from the table. The same values of $T_{0}, \alpha_{k}$ and $\mu_{k, 0}$ used for the multi-species calculations are provided to the look-up table model. The reference heat capacity and viscosity are then obtained using the '2/3-1/3' formalism where temperature $T_{g}$ in Eq. (22) is obtained from Eq. (3):

$$
\mu^{\text {evap }}=\sum_{k=1}^{6} \frac{X_{k}^{\text {evap }} \mu_{k}^{\text {evap }}}{\sum_{l=1}^{N_{s}} X_{l}^{\text {evap }} \phi_{k l}^{\text {evap }}}, \quad c_{p}^{\text {evap }}=\sum_{k=1}^{6} c_{p}^{k} Y_{k}^{\text {evap }} .
$$

\section{The MERCATO benchmark}

\subsection{Experimental configuration}

The experimental MERCATO benchmark [12] studied at the ONERA Fauga-Mauzac has been retained in this work since it is representative of the two-phase flow produced by an industrial swirl turbojet injection system. As represented in Fig. 1(a), it consists of a plenum, a pressure-swirl fuel injector provided by Turbomeca (Safran group), a combustion chamber and an exhaust section. Air is injected into the plenum at ambient conditions $(P=1 \mathrm{~atm}$, $\left.T=285 \mathrm{~K}, \dot{m}_{a i r}=0.035 \mathrm{~kg} / \mathrm{s}\right)$, then reaches the injector where it is swirled at a Swirler number $S w=0.65$ and finally enters the combustion chamber. Liquid fuel is also injected into the combustion chamber by a fuel atomizer at ambient temperature $\left(T_{l}=285 \mathrm{~K}, \dot{m}_{l}=\right.$ $0.00225 \mathrm{~kg} / \mathrm{s}$ ). The liquid jet is atomized, evaporates into the chamber and finally mixes with air. A swirling two-phase flame, classically characterized by a central recirculation zone and two outer recirculation zones, is stabilized in the combustion chamber. A Precessing Vortex Core (PVC) motion has been detected under non-reactive conditions [12]. Laser Doppler Anemometry/Phase Doppler Anemometry (LDA/PDA) measurements are available for gas velocity as well as droplet velocity at four different axial distances from the diffuser exit, $x=10,26,56$ and $116 \mathrm{~mm}$. Droplet diameter profiles are also available at $x=10,26,56$ and $86 \mathrm{~mm}$.

\subsection{Numerical setup}

The numerical configuration reproduces the experiment including the whole exhaust section (not shown). The computational domain has been meshed using approximately 11 million tetrahedral cells which are refined in the region of interest, i.e. the injection and the reaction zones, represented in Fig. 1(b). The grid quality has been verified by simulating the reactive case with the tabulated technique also on a finer mesh (approximately 20 million tetrahedral cells). Grid convergence results are presented in Section 4.3.

Large Eddy Simulations of the MERCATO configuration are performed with the AVBP solver. The non-reactive case has already been numerically studied by Senoner et al. [13] and 


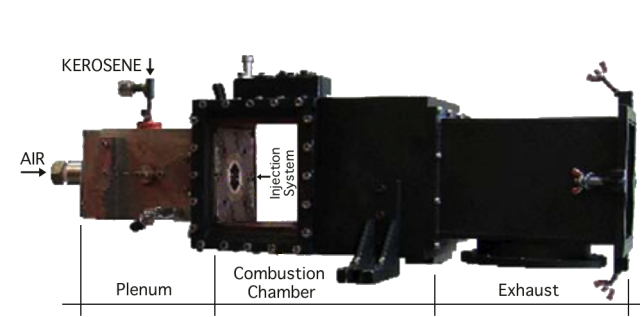

(a) The experimental setup [12]

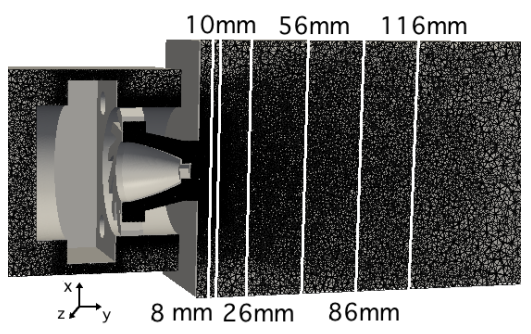

(b) Computational half-domain mesh [10] The measurement sections are indicated.

Fig. 1: MERCATO configuration.

Sanjosé et al. [14] for different operating conditions (i.e, air was preheated at temperature $T=473 \mathrm{~K}$ before being injected into the plenum). Moreover, a previous LES of the nonreactive case corresponding to the reactive case studied here has also been performed by Franzelli et al. [10]. A good agreement between numerical and experimental results was achieved for the cold cases in the previous calculations confirming the validity of the twophase modeling strategy, even if it has also been shown that the monodisperse assumption limit the ability of the method to capture the outer part of the spray [15].

Here, the same setup used by Senoner et al. [13] and Franzelli et al. [10] is applied for the reactive calculations. The numerical integration is performed using a third-order in time and space Taylor-Galerkin scheme [44] on both liquid and gas phases. The Navier-Stokes Characteristic Boundary Conditions (NSCBC) [45] are used to impose gas inlet and outlet boundary conditions. Liquid injection is imposed with the FIMUR (Fuel Injection Method by Upstream Reconstruction) methodology and a droplet diameter at injection $d_{l}^{0}=43 \mu \mathrm{m}$. The FIMUR methodology generates boundary conditions for a disperse phase modeling. It avoids the simulation of the primary atomization by means of empirical laws and selfsimilarity assumptions ${ }^{6}$ as described in [14]. All walls are treated with an adiabatic no-slip condition.

The same numerical setup is used for both calculations. The 24-species mechanism [11] is used to build the look-up table. Laminar freely-propagating premixed flames at the operating conditions for the gas phase injection of the MERCATO burner (i.e. fresh gas temperature $T=285 \mathrm{~K}$, pressure $P=1 \mathrm{~atm}$ ) have been calculated using the REGATH code [46] for four hundred different equivalence ratios (i.e. mixture fraction) within the flammability limits under the unity Lewis number assumption. The look-up table is uniformly discretized by 100 points for the progress variable $Y_{c}$ and 400 points for the mixture fraction $Y_{z}$. Due to the high number of species transported in the multi-species chemistry case, its computational cost is eight times higher than the tabulated case.

\subsection{Numerical validation}

The MERCATO burner benchmark has been simulated for about $180 \mathrm{~ms}$. In order to guarantee the statistical convergence of the flow, the mean flow is obtained by averaging the results for the last $80 \mathrm{~ms}$, corresponding to more than 10 convective times. Under reactive

\footnotetext{
6 The nozzle geometry has been modified according to this model.
} 
conditions, the swirled flame stabilizes in the combustion chamber with both chemical descriptions. In order to globally characterize the flame, the averaged temperature field on the axial plane obtained with the detailed 24-species scheme is shown in Fig. 2, together with the zero iso-contour of axial velocity, allowing the identification of the recirculation zones, characteristics of swirled flames. The reactive flow presents a central inner recirculation zone (IRZ), where burnt gases are transported back towards the nozzle by the inner reverse flow and an outer recirculation zone (ORZ).

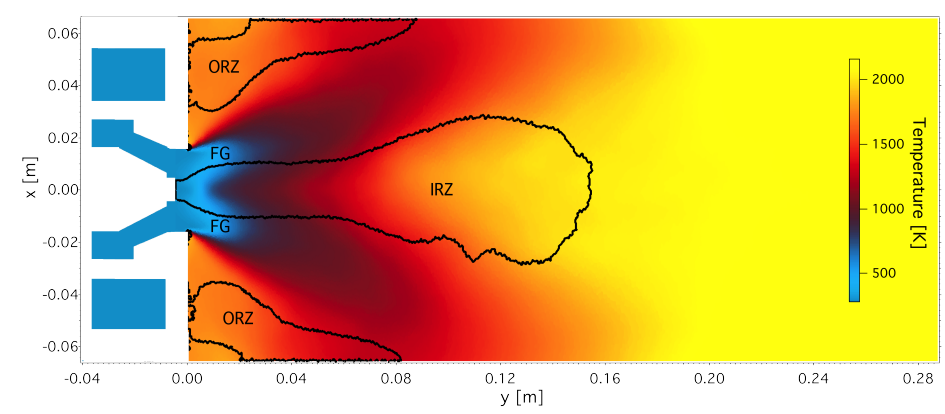

Fig. 2: Mean gas temperature distribution. In order to localize the recirculation zones black isocontour of zero axial gas velocity is added.

Numerical results obtained by both multi-species and tabulated approches on the reference 11 million-cell mesh are compared to experimental data (symbols) in Figs. 3-4 by black continuous and blue dashed lines, respectively. Results on the finer 20 million-cell mesh using the tabulation approach are also added in red dotted lines to verify the grid convergence.

Numerical data for the mean axial velocity shown in Fig. 3(a) are in agreement with experiments. The penetration of fresh air, identified by a positive axial velocity in planes $y=10 \mathrm{~mm}$ and $y=26 \mathrm{~mm}$, is correctly reproduced along the chamber, even if the maximum velocity value and the opening of the gas jet are slightly underestimated.

Concerning the fluctuation of the axial velocity represented in Fig. 3(b), discrepancies between numerical and experimental results are detected mainly at $x=10 \mathrm{~mm}$ for the reactive case whereas the behavior is correctly reproduced on the other measurement planes. At $x=10 \mathrm{~mm}$, the experimental velocity fluctuations are the highest in the IRZ and are surprisingly low in the fresh gas (FG) region where the axial velocity is high, in contrast with numerical results which localize the highest fluctuations in the FG and reaction zone regions.

Concerning the liquid phase, radial profiles of Sauter mean droplet diameter $d_{32}$ have been measured at four axial positions $x=10,26,56$ and $86 \mathrm{~mm}$ and are compared in Fig. 4(a) to the LES results. The measurements performed downstream in the ORZ reveal the presence of big droplets. These large droplets cannot be captured by the simulation as they are strongly linked to the polydisperse character of the spray, as already observed by Senoner et al. [13] and Vie et al. [15] under non-reactive conditions. In [47], the experimental droplet size distribution varies between $2 \mu \mathrm{m}$ and $150 \mu \mathrm{m}$ whereas the mean diameter is $44 \mu \mathrm{m}$. In the monodisperse LES framework, only the mean diameter is solved and big droplets dynamics cannot be captured. This phenomenon is even more pronounced in our simu- 


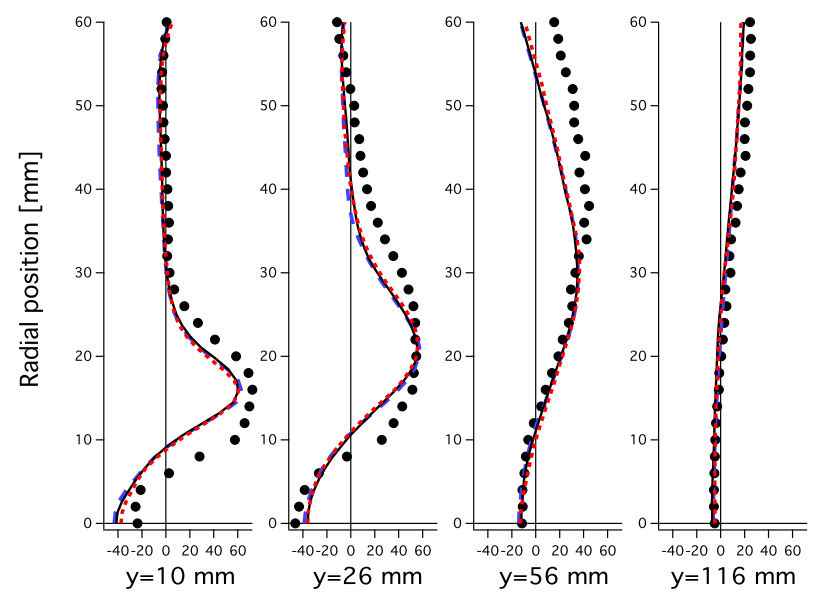

(a) Mean gas axial velocity $(\mathrm{m} / \mathrm{s})$

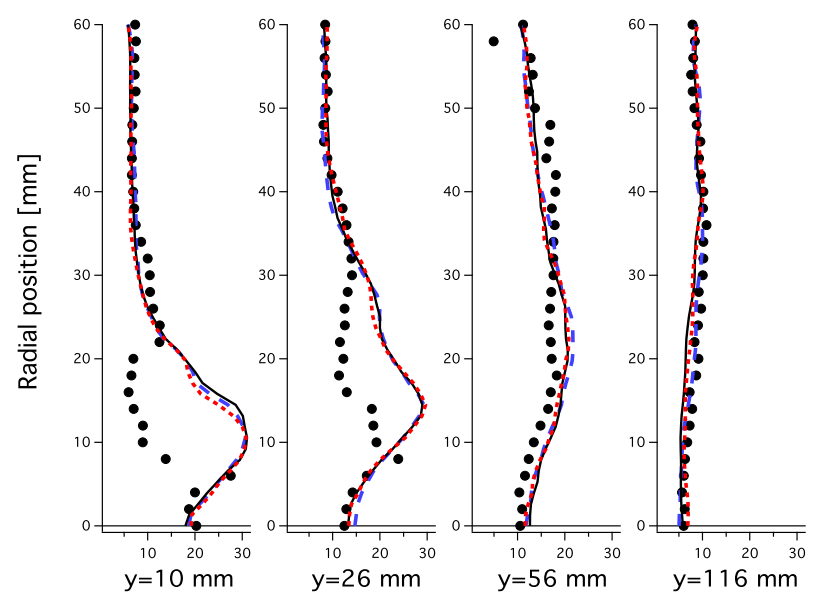

(b) RMS of the gas axial velocity $(\mathrm{m} / \mathrm{s})$

Fig. 3: Mean and rms gas quantities as a function of radial position for different distances from the injector. Numerical results (lines) are compared to experimental data (symbols). Numerical results have been obtained on the 11 million-cell grid with the multi-species (black continous line) and the tabulated (blue dashed line) descriptions and on the 20 million-cell grid with the tabulated technique (red dotted line).

lations, since under reactive conditions the smallest droplets evaporate quickly while the most-inertial largest ones accumulate in the ORZ, where discrepancies are the highest.

The mean liquid axial velocity profiles shown in Fig. 4(b) are correctly predicted even if their maximum values are underestimated. As discussed in [48], neglecting the random uncorrelated energy ${ }^{7}$ changes the balance of momentum for the disperse phase for inertial droplets so that the droplet velocity fluctuations, represented in Fig. 4(c), are underestimated.

\footnotetext{
7 The random uncorrelated energy corresponds to the droplets velocity dispersion around the local mean velocity [49].
} 


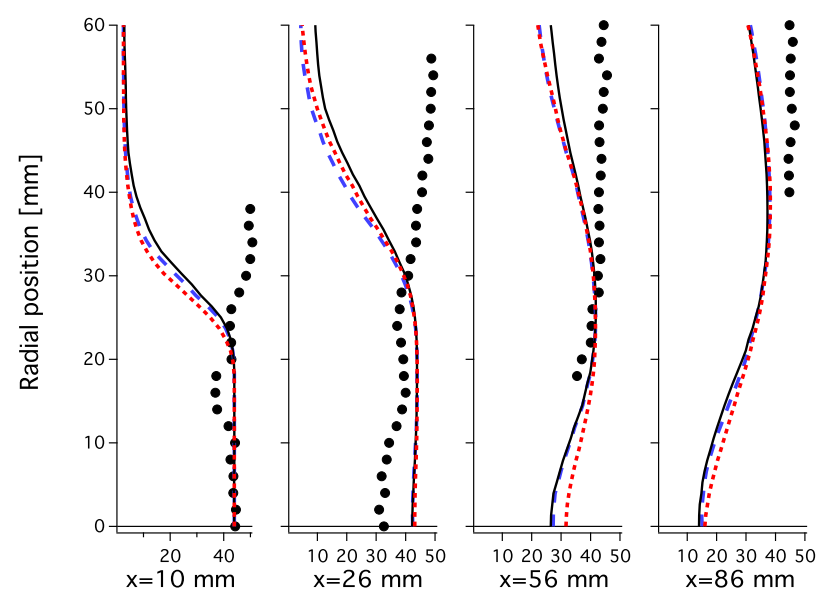

(a) Mean Sauter droplet diameter $(\mu m)$

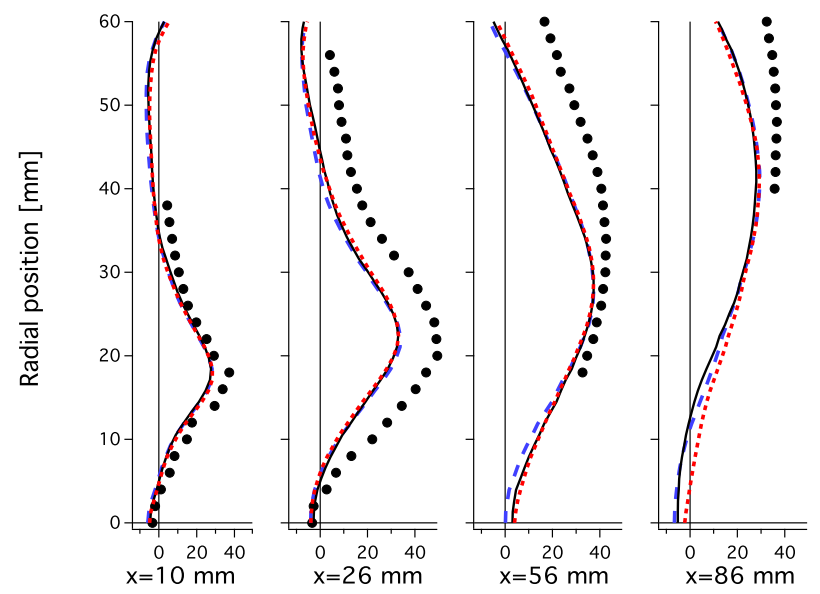

(b) Mean liquid axial velocity $(\mathrm{m} / \mathrm{s})$

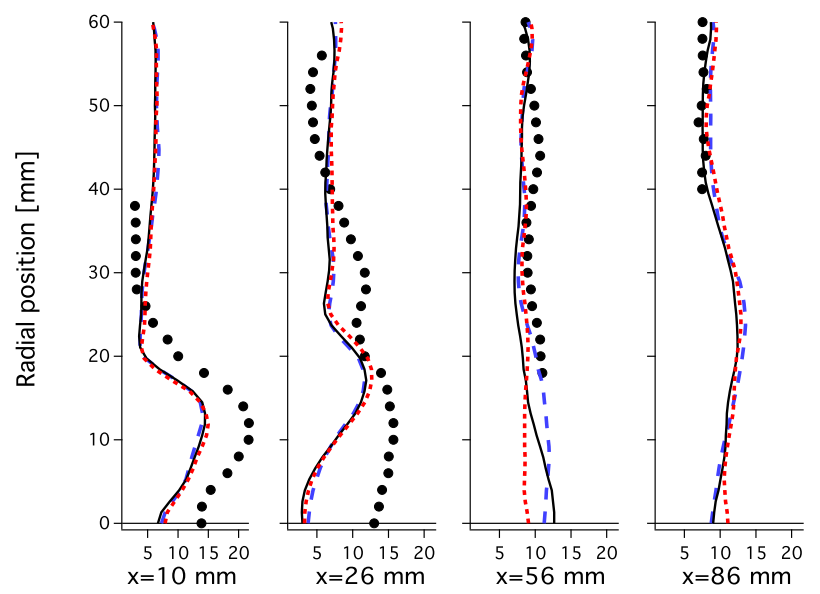

(c) RMS of the liquid axial velocity $(\mathrm{m} / \mathrm{s})$

Fig. 4: Mean and RMS liquid quantities as a function of radial position for different distances from the injector. Numerical results (lines) are compared to experimental data (symbols). Numerical results have been obtained on the 11 million-cell grid with the multispecies (black continous line) and the tabulated (blue dashed line) descriptions and on the 20 million-cell grid with the tabulated technique (red dotted line). 
Globally, the agreement between the two numerical approaches is satisfactory when looking at the experimentally-observed quantities. The liquid mono-dispersed mono-kinetic assumption for the liquid phase affects the spray description but still represents a good compromise between accuracy and computational cost, necessary for the 24-species computation. Moreover, the tabulated method seems to be a good candidate for LES of swirled spray flames at reduced CPU costs. Only small discrepancies are detected for the mean droplet diameter in the outer recirculation zone close to the wall. Negligible differences localized in the inner recirculation zone are found when comparing the FPI results on the two considered grids so that it can be assumed that the grid convergence is already reached on the 11 million-cell grid. Indeed, results obtained on the coarse grid will be presented in the following.

\section{Flame characterization}

The flame structure and the complex dynamics of the swirled spray flame MERCATO were not investigated experimentally. However, this can be done here numerically by looking at the 24-species chemistry results as a reference. Such kinetical description accounts for the main chemical pathways so that the chemical processes are accurately predicted (and not modeled as in the case of tabulated techniques or fitted chemistries). In the following, the effect of the evaporation-mixture-combustion interactions is analyzed in terms of flame structure and dynamics.

\subsection{Flame structure}

The complex nature of the spray flame structure is characterized in this section by analyzing the multi-species chemistry results. The instantaneous fields are presented in Figs. 5 and 6. In Fig.5(a), liquid volume fraction isocontours colored by the droplet number density are presented together with the instantaneous temperature field. The high-evaporation regions are also identified by the black zones. The spray generates thin structures because of the swirling motion of the gas phase and the turbulence. It can be observed that the spray slightly evaporates in the fresh gas zone, due to its low temperature, and reaches the IRZ/FG zone, close to the flame front. The high evaporation zones are then located in the pre-heating region of the flame, where the temperature increases due to diffusion from the burnt gases of IRZ and ORZ. The strong evaporation process can also be identified by the presence of gaseous fuel mass fraction (see. Fig.5(b)). Then, since enough fuel is provided to the gaseous mixture, the combustion occurs in a primary reaction zone, identified in Fig.5(b) by the fuel consumption rate isocontours in red, which envelopes the high-evaporation zones. The heat provided to the burnt gases located in IRZ and ORZ is enough to sustain evaporation and, consequently, combustion.

A part of the liquid volume fraction goes through the flame front and reaches the wall. This is possible because droplets of $44 \mu \mathrm{m}$ diameter have a large vaporization time compared to their convective time from the injection to the wall. As shown in Figs. 5(c)-5(d), the droplet temperature is the highest close to the wall, where droplet diameter is still larger than $30 \mu \mathrm{m}$. It shows that the vaporization process is not complete after going through the primary reaction zone (about $30 \%$ of the liquid mass is still not evaporated). This leads to the presence of a secondary reaction zone. It can be observed in Fig. 6(a) that part of the evaporation occurs in the burnt gases downstream the flame front, so that the mixture equivalence 


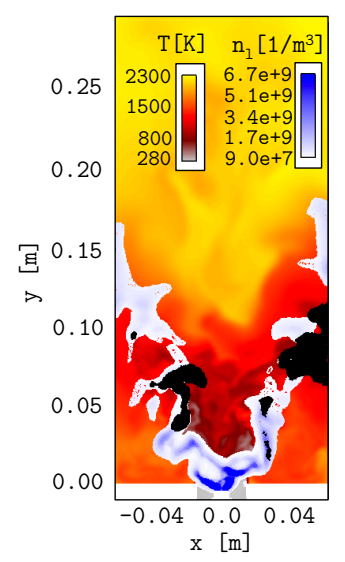

(a) Temperature field and liquid volume fraction isocontours colored by the droplet number density. The highevaporation zones are identified in black.

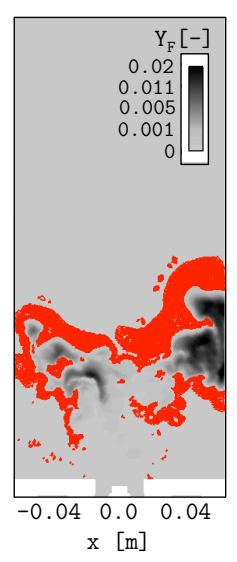

(b) Fuel mass fraction field. The primary reaction zone is identified by red isocontours of fuel consumption rate.

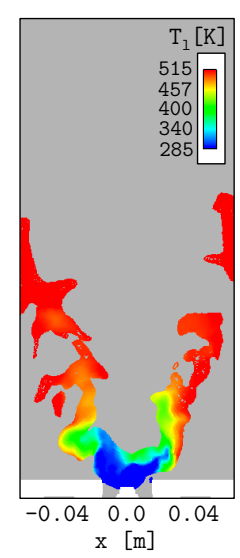

(c) Liquid volume fraction colored by the liquid temperature.

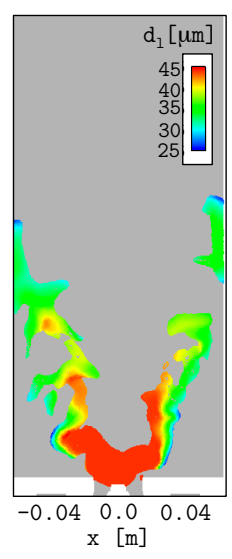

(d) Liquid volume fraction isocontours colored by the droplet diameter.

Fig. 5: Characterization of the primary reaction zone.

ratio increases. In this zone, small amounts of gaseous fuel immediately reacts with the burnt gases due to their temperature leading to the formation of high $\mathrm{CO}$ concentration pockets close to the wall at the end of the chamber (Fig. 6(b)). The presence of a secondary reaction zone is clearly identified by looking to the $\mathrm{CO}$ formation (positive in blue) and destruction (negative in red) zones in Fig. 6(c). A first formation zone, followed by a destruction region, is found in the primary reaction zone. The $\mathrm{CO}$ concentration assumes a constant equilibrium value before reaching the secondary zone, where high values of $\mathrm{CO}$ are found in the second $\mathrm{CO}$ formation zone. Due to the recombination processes, the CO mass fraction then slowly decreases in the second destruction zone towards its equilibrium value.

The mean flame front localisation and structure, presented in Fig. 7, reflect the observed processes. Blue iso-contour of zero axial gas velocity identifies the IRZ and ORZ regions while a black isocontour of heat release, corresponding to the $10 \%$ of its maximum value, is used to localize the reaction zone in Fig. 7(a). In Fig. 7(b), iso-contours of the liquid volume fraction, indicating the spray position, are colored by the liquid temperature (left), whereas the $\mathrm{CO}$ formation/consumption zones are identified by blue/red isocontours of positive/negative $\mathrm{CO}$ production rate (isocontours correspond to $10 \%$ of maximum value) together with the $\mathrm{CO}$ mass fraction field (right). The spray and the fresh air are localized in the fresh gas zone. The flame is stabilized in the FG/ORZ layer close to the injection and downstream the spray where a sufficiently rich mixture is obtained thanks to evaporation.

Close to the injection, the IRZ is characterized by a moderate temperature (Fig.7(a)right) so that the evaporation is relatively slow and the mixture equivalence ratio slowly increases (Fig.7(a)-left). On the contrary, the gaseous mixture in the ORZ is characterized by higher temperature and equivalence ratio. This is due to the fact that both gas and liquid phases are characterized by a small axial velocity (cfr. Figs. 3(a) and 4(b)), i.e. a longer convective time characterizes the ORZ compared to the IRZ. Indeed, spray droplets in ORZ are heated up more (as proven by the field of the liquid temperature in Fig. 7(b)-left). As 


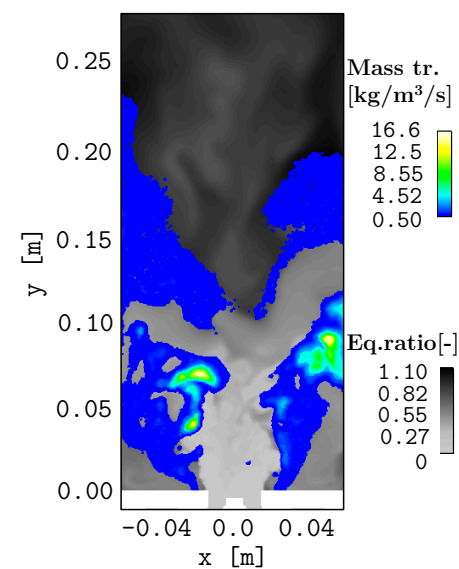

(a) Equivalence ratio field. The evaporation zones, colored by the vaporization rate, are added.

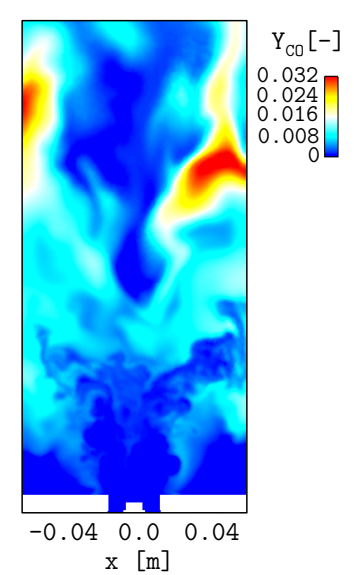

(b) CO mass fraction field.

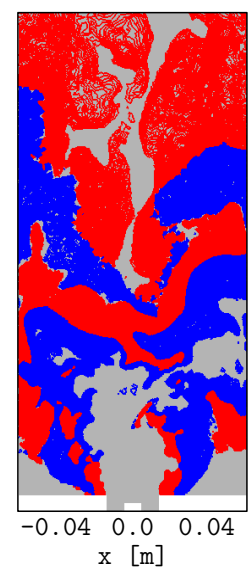

(c) $\mathrm{CO}$ formation and consumption zones are identified in blue and red, respectively.

Fig. 6: Characterization of the secondary reaction zone by looking to the instantaneous flame structure.

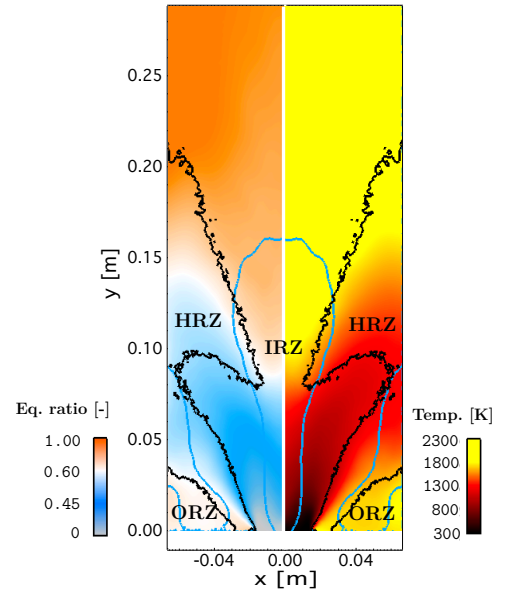

(a) Equivalence ratio (left) and temperature (b) Iso-contours of the liquid volume frac(right) fields. Blue isocontour of zero axial gas tion colored by the liquid temperature (left) velocity and black isocontour of heat released and $\mathrm{CO}$ mass fraction field (right). $\mathrm{CO}$ forare added.

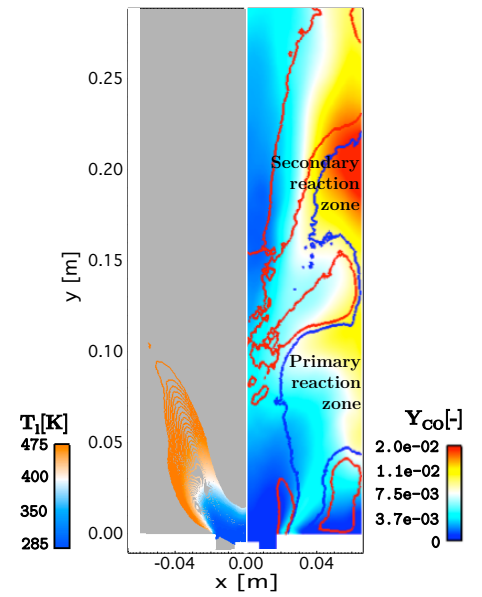
mation and consumption zones are identified in blue and red, respectively.

Fig. 7: Time-averaged flame structure.

a consequence, the evaporation process is faster so that a higher equivalence ratio characterizes the ORZ close to the injection compared to the IRZ. The reaction zone, identified 
by the heat release zone (HRZ) in Fig. 7(a), is localized in the layer located between the fresh gas injection and the ORZ. Being characterized by a long convective time, the gaseous mixture reaches the equilibrium state in the ORZ, so that the high temperature enhances the evaporation process and contributes to the flame stabilization.

Downstream, the reaction zone is located where the mixture is rich enough to sustain combustion. The presence of primary and secondary reaction zones cannot be identified by looking to the temperature field, since the contribution of the secondary reaction zone to the total heat release is negligible. However, its presence and its effect on emission distributions are evident when looking to the mean field of $\mathrm{CO}$ mass fraction (Fig.7(b)-right), which presents two pockets of high $\mathrm{CO}$ concentration close to the primary and secondary reaction zones. This complex flame structure is due to an intricate coupling between evaporation, mixing and combustion governing the stabilization location of the flame front. Moreover, emissions seem highly sensitive to the inhomogeneities of the mixture fraction field, as observed for the secondary reaction zone due to fuel evaporation downstream the primary flame front. The capability of the tabulated technique to reproduce such an intricate flame structure will be discussed in Section 6.

\subsection{Flame dynamics}

Not only the flame-spray-mixture interactions affect the flame structure and its stabilisation process, but they also govern the flame dynamics. Even if the mean flow is symmetrical in space, the instantaneous flame structure strongly varies in space with time. The swirled flow is characterized by a swirling time $\tau^{\text {swirling }}=\frac{2 \pi}{\omega} \approx 10 \mathrm{~ms}$ based on the averaged orthoradial gas frequency $\omega$ at the chamber inlet. The liquid droplet trajectories follow this swirling motion, but at the same time the spray behaviour is also affected by another flow motion: the precessing vortex core. In this configuration, the frequency of this hydrodynamics instability is measured at $f_{P V C}=1120 \mathrm{~Hz}$ using pressure probes, which is in agreement with experimental observations. In Fig. 8, four instantaneous snapshots of pressure and liquid volume fraction isosurfaces are represented at equidistant times in the precessing period of the PVC. The liquid volume fraction $\alpha_{l}$ follows the helicoidal motion of the PVC since droplets are ejected from the PVC by their inertia. The liquid volume fraction presents an oscillating behaviour at the same frequency as the PVC, but with a phase shift due to its high Stokes number $\left(\mathrm{St}_{P V C} \approx 10\right)$.

As a consequence of this motion, gaseous kerosene pockets are created by the evaporation process close to the high liquid concentration region following the PVC. The gas temperature distribution on the first measurement plane $(x=8 \mathrm{~mm})$ is presented in Fig. 9 for one precessing period of the PVC. Iso-contours of the vaporization rate, of the pressure and of the liquid volume fraction are also superimposed. It is shown that the high volume fraction zone is followed by a vaporization zone in this helicoidal motion while high vaporization is also experienced by the droplets in the ORZ. The temperature field (indicative of the flame front position) is also affected by this motion, confirming this strong coupling between PVC, vaporization and flame. The spray-PVC interaction has then a very strong impact on the flame front that moves accordingly with the PVC, proving that the dynamics of this flame is mainly governed by the coupling between evaporation, mixing and chemical processes. 


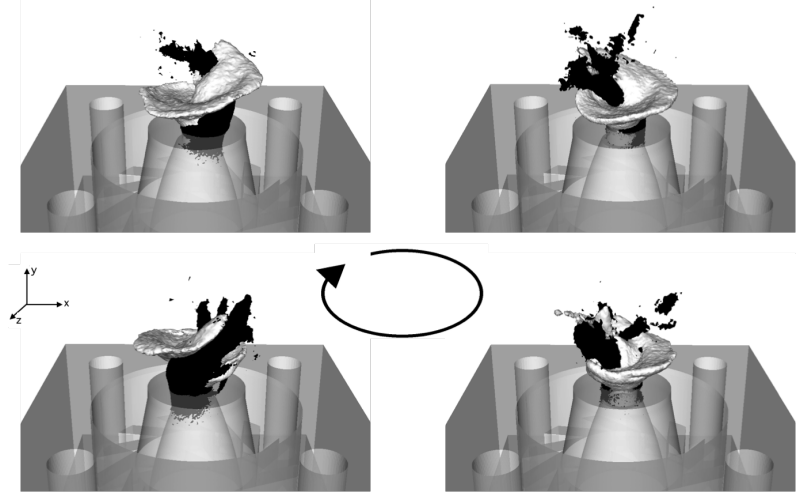

Fig. 8: Instantaneous pressure iso-surface in black $(P=0.10 \mathrm{MPa})$ and liquid volume fraction iso-surface in white $\left(\alpha_{l}=2.4 \cdot 10^{-4}\right)$. Four instantaneous solutions are considered at equidistant times for one period of the PVC.

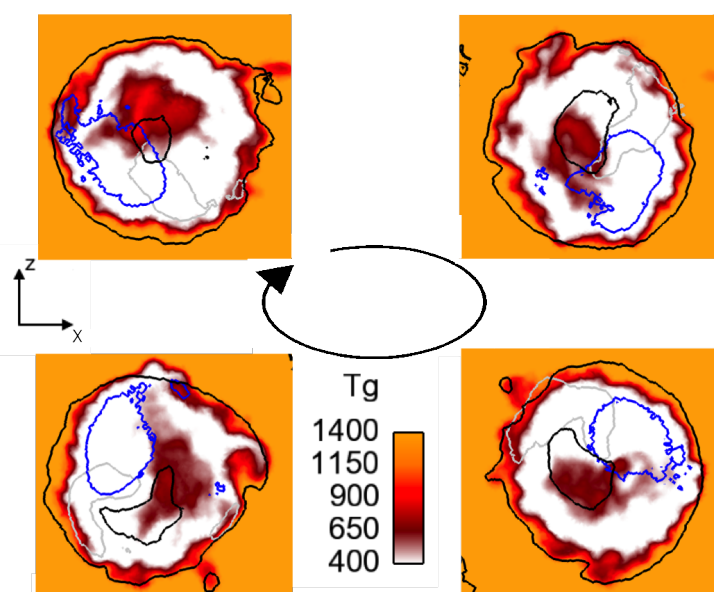

Fig. 9: Pressure, liquid volume fraction and mass transfer iso-contours are represented at four instants of one period of the PVC on the first measurement plane $(x=8 \mathrm{~mm})$, for $p=$ $0.995 \mathrm{MPa}$ (blue), $\alpha_{l}=2 . \cdot 10^{-4}$ (grey) and $\dot{m}=0.05 \mathrm{~kg} \cdot \mathrm{m}^{-3} . \mathrm{s}^{-1}$ (black) respectively. For $\dot{m}$, the maximum values are reached outside the external contour and inside the internal contour. The colormap denotes the instantaneous gas temperature distribution saturated at $1400 \mathrm{~K}$.

\section{Evaluation of the FPI tabulation method for swirled spray flames}

In Section 5, it has been discussed that the coupling between evaporation, mixing and combustion governs the flame dynamics, stabilisation and structure. The objective of this section is to verify if the tabulation technique is able to reproduce the above flame features for a reduced computation cost. In this sense, the 24 -species results is considered as the reference 
case. Such strategy has been already successfully applied to evaluate reduced semi-global mechanisms for turbulent purely gaseous flames [38].

The flame dynamics discussed in Section 5.2 was already retrieved by the FPI tabulation method [10], proving its capability in reproducing the macroscopic flame-spray-turbulence interactions. In the present work, we focus on the ability of the tabulation method to reproduce the flame structure and pollutant emissions.

Time-averaged results for the flame structure are presented in Fig. 10 for both the 24species (left) and the FPI look-up table technique (right). The flame length is slightly underestimated by the FPI method, but its shape and flame structure are correctly reproduced in Fig. 10(a). Some discrepancies are observed close to the external wall where the flame interacts more with the wall in the 24-species case than in the FPI case. This behaviour has already been observed in a LES of a turbulent perfectly premixed gaseous flame [50]. Being based on laminar freely-propagation premixed flames, the FPI method seems not to reproduce accurately the sensitivity of the flame to strain rate. The consumption speed is expected to decrease with the strain rate [35]. The FPI technique mispredicts the strain rate effect on the consumption speed, leading to its overestimation. Consequently, the flame core predicted by the FPI technique is smaller since the fuel is consumed more rapidly than with the 24-species description. The mixture fraction and temperature fields of Figs. 10(b) and 10 (c) reflect the $Y_{c}$ behaviour, being strongly related to each other through the evaporation process.

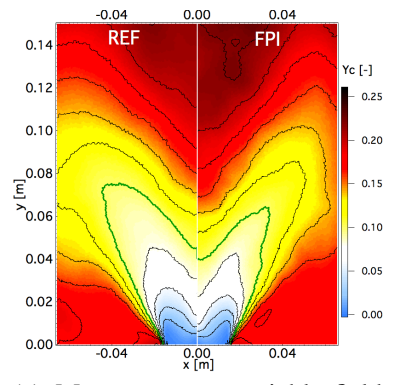

(a) Mean progress variable field and isocontours. The green isocontour corresponds to $Y_{c}=0.1$.

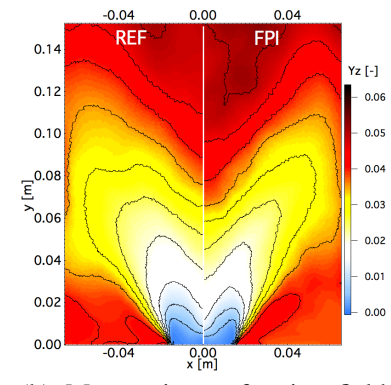

(b) Mean mixture fraction field and isocontours.

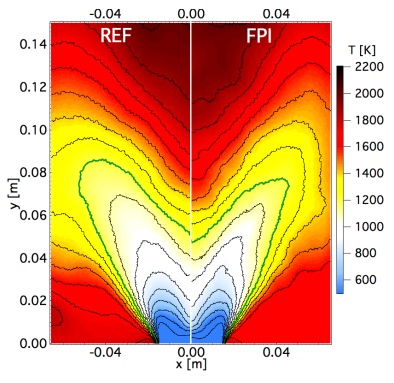

(c) Mean temperature field and isocontours. The green iso-contour corresponds to $T=1200 \mathrm{~K}$.

Fig. 10: Time averaged flame structure. Results with the tabulated technique (right half) are compared to the 24-species results (left half) for progress variable (a), mixture fraction (b) and temperature (c).

Some discrepancies are also observed in the ORZ close to the injection where the temperature predicted by the multi-species description is slightly higher than the FPI method results for $y<0.02 \mathrm{~m}$. However, the overall agreement is satisfactory as shown by the radial profiles in Fig. 11, proving that the use of the TTC approach is accurate enough to account for the heat transfer between gas and liquid phases in the temperature field without the need for an additional dimension of the look-up table.

Results for the liquid volume fraction presented in Fig. 12 confirm the ability of the FPI method to reproduce the liquid phase characteristics, which was not trivial since a correct prediction of the strong coupling between spray, mixture fraction and flame temperature is needed to predict spray combustion. 


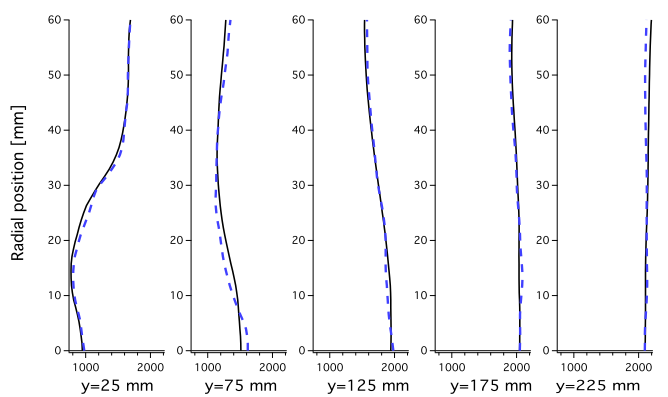

Fig. 11: Temperature profile as a function of radial position for different distances from the injector. Numerical results with the 24-species scheme (black continuous line) are compared to the tabulated description (blue dashed line).

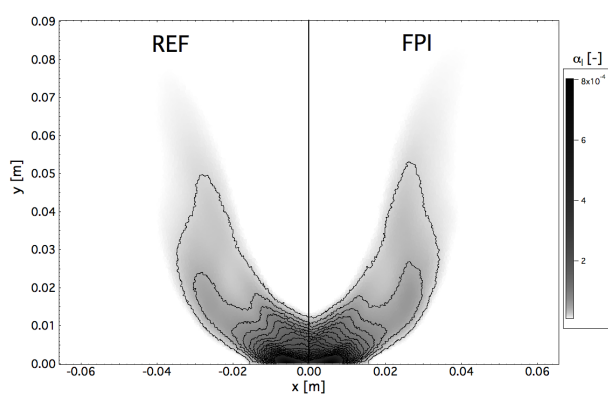

Fig. 12: Time averaged liquid volume fraction. Results with the tabulated technique (FPI, right half) are compared to the multi-species results (REF, left half).

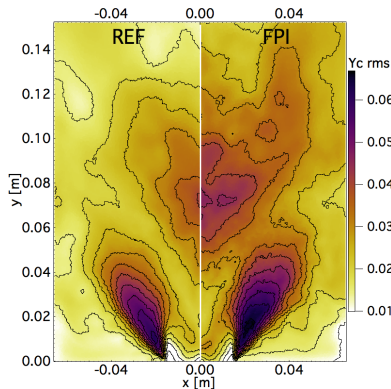

(a) Progress variable RMS

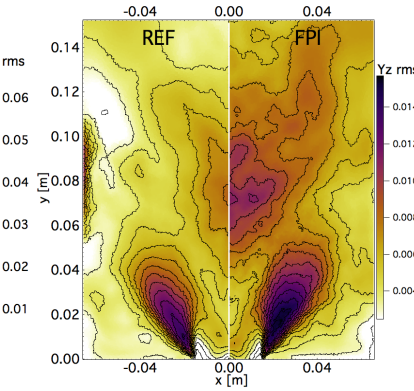

(b) Mixture fraction RMS

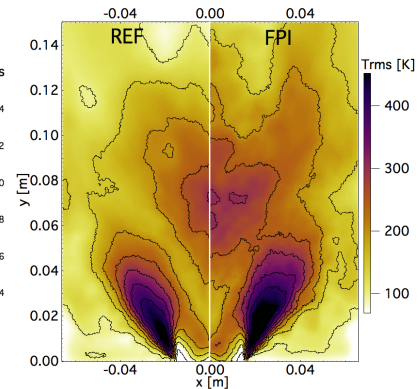

(c) Temperature RMS.

Fig. 13: Fluctuations of the flame structure. Results with the tabulated technique (FPI, right half) are compared to the 24-species results (REF, left half).

To complete the analysis on the global flame structure, the fluctuation field of $Y_{c}, Y_{z}$ and temperature are shown in Fig. 13. Once again, the overall agreement is good, even if slightly higher fluctuations are observed in the IRZ for the FPI case.

Results for $\mathrm{CO}_{2}$ and $\mathrm{CO}$ mass fractions are presented in Figs. 14(a) and 14(b), respectively. The $\mathrm{CO}_{2}$ field reflects the results discussed for the temperature. The presence of an 


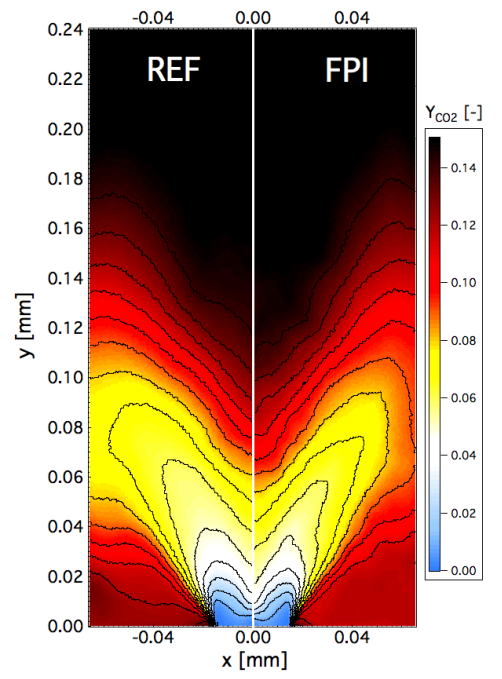

(a) Mean $\mathrm{CO} 2$ mass fraction.

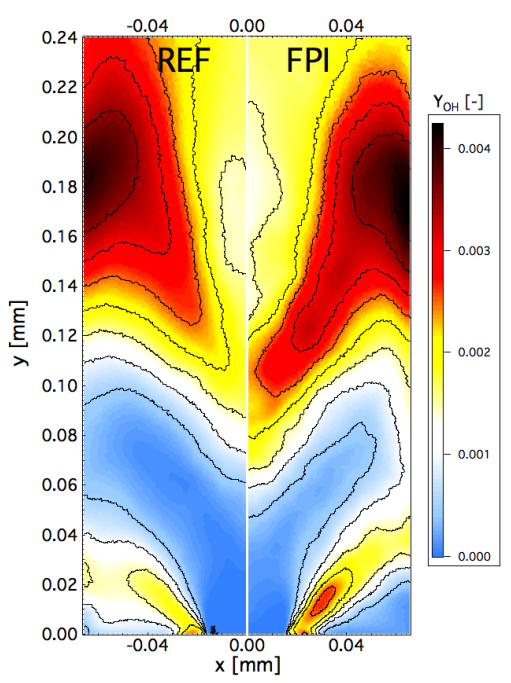

(c) Mean $\mathrm{OH}$ mass fraction.

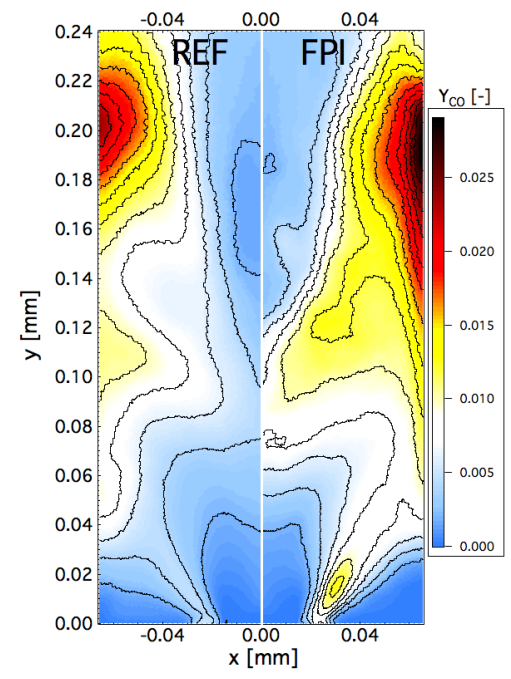

(b) Mean CO mass fraction.

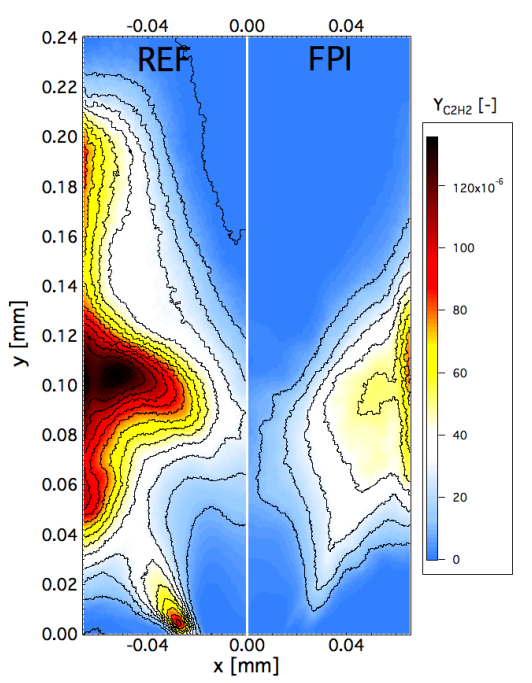

(d) Mean $\mathrm{C} 2 \mathrm{H} 2$ mass fraction.

Fig. 14: Time averaged fields for pollutants and intermediate species. Results with the tabulated technique (FPI, right half) are compared to the 24-species results (REF, left half).

high CO concentration region is correctly reproduced by the FPI approach. HA deeper comparison of the $\mathrm{CO}$ profiles presented in Fig.15(a) reveals significant discrepancies in the HRZ, where the CO mass fraction is overestimated by the FPI method. As deduced by looking to the RMS results in Fig. 13 and the mixture fraction field in Fig. 10(b), these zones are characterized by the presence of strong flow fluctuations, i.e. high strain rate, and of a high gradient of $Y_{z}$, i.e. highly non-homogeneous mixture, whose effects on the $\mathrm{CO}$ concentration are not accurately accounted for by the FPI method. The same conclusions hold for $\mathrm{OH}$ 
mass fraction, presented in Figs. 14(c).

Some discrepancies are also detected for the second reaction layer for CO. The FPI method
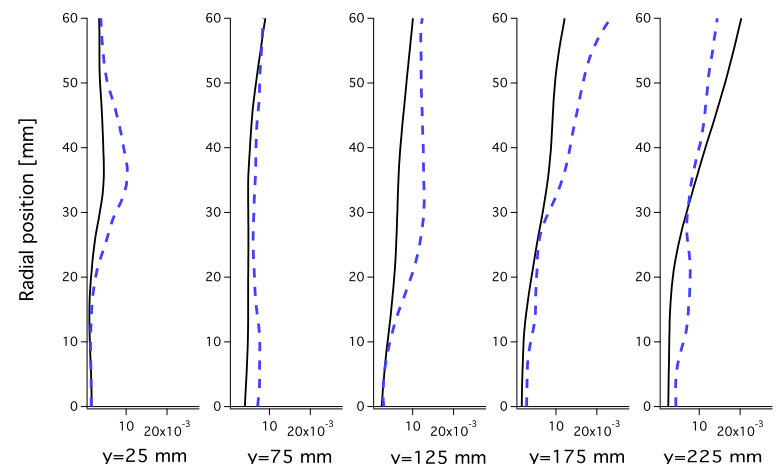

(a) $\mathrm{CO}$ mass fraction
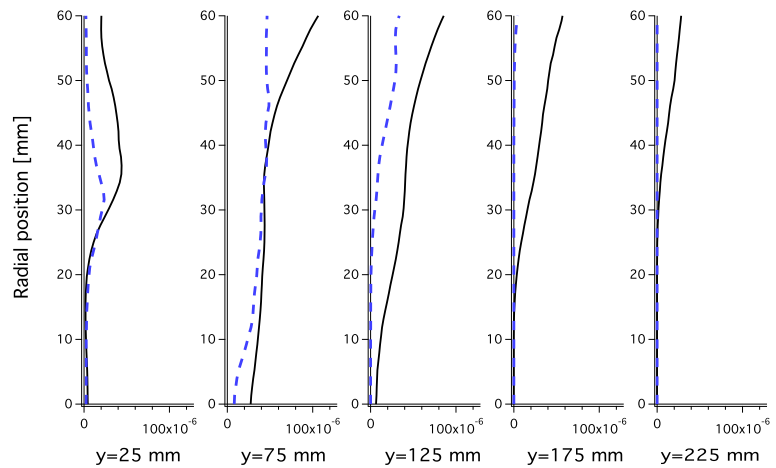

(b) $\mathrm{C} 2 \mathrm{H} 2$ mass fraction

Fig. 15: Mean species mass fraction as a function of radial position for different distances from the injector. Numerical results with the 24-species scheme (black continuous line) are compared to the tabulated description (blue dashed line). Letters identified the regions represented in Fig. 7.

seems to have some difficulties in reproducing it the recombination process due to the secondary evaporation in the burnt gases of this region. LES of the MERCATO bench and laminar counterflow spray flames simulations (Appendix A) lead to similar conclusions. The FPI method correctly reproduces the main features of spray flame structure, such as the temperature, the liquid volume fraction and the mixture fraction. However, inaccuracies are detected in pollutants such as CO. Non-premixed-like combustion, dilution with burnt gases and strain rate effects are, indeed, not accounted for in the tabulated manifold affecting the emission prediction. Such limits are even more pronounced for minor species and radicals. As an example, acetylene prediction is represented in Fig.14(d). This species can be an indication of the ability of the FPI model to describe the complex nature of the chemical processes leading to intermediates, minor species and radicals. It should be reminded that in the context of soot prediction a correct description of the acetylene and of the precur- 
sors, which are strongly sensitive to strain rate [51], is essential [52]. Even if the acetylene localization is qualitatively predicted, the tabulated technique significantly underestimates its concentration (Fig. 15(b)). In this sense, the prediction of intermediates and radicals in a swirled spray flame provided by a tabulation method based on one single archetypical flamelet is only qualitative, even if they are correctly localized. Even if the FPI method provides a good prediction of the global structure of a swirled spray flame for a reduced computational cost, more sophisticated techniques are required to produce quantitative predictions of pollutants $[1,38]$.

\section{Conclusion}

LES of the MERCATO experimental benchmark has been performed using a detailed chemical description accounting for 24-species to study the behavior of an industrial swirled twophase injection system. Numerical results have been compared to the experimental flow in terms of mean and fluctuations of axial velocity of both gas and liquid phases as well as droplet diameter profiles. The strong interaction between PVC and heat release in spray swirled flames has already been identified in other spray configurations both experimentally and numerically $[53,54]$. While this phenomenon was not investigated experimentally in the MERCATO benchmark, the dynamics of the spray swirled flame have been here numerically recognized and characterized. Moreover, the effect of evaporation-mixing-combustion coupling on the flame structure has been analyzed. Additionally, a second LES using the FPI tabulated chemistry has been performed to investigate its ability to correctly reproduce the complex spray flame structure for a reduced computational cost. It has been shown that tabulated chemistry methods allow the numerical investigation of swirled industrial spray flames, at least for the prediction of global flame behavior, with a cost which is eight times smaller than the considered multi-species description.

The present LES strategy for two-phase turbulent reacting flows has two limitations that will be addressed in future simulations. The first one is due to the spray monodisperse assumption, which cannot accurately predict equivalence ratio stratification. An attractive solution, that will not affect the combustion modeling strategy, is to implement a polydisperse Eulerian approach [15]. The second limitation is related to the prediction of intermediate species and pollutants which is extremely sensitive to the model used to describe the chemical structure. Indeed, numerical investigations of laminar counterflow spray flames [21] highlight situations where the chemical structure of spray flame fronts deviates from standard premixed gaseous flame archetypes. However, the results obtained on the LES with the FPI method are encouraging the development and use of more sophisticated tabulated models for pollutant prediction of spray combustion.

\section{Acknowledgment}

This work was granted access to the HPC resources of CINES under the allocation $\times 20152 \mathrm{~b} 6172$ made by GENCI (Grand Equipement National de Calcul Intensif). CERFACS is also gratefully acknowledged for providing the AVBP code and the meshes for our computations.

\section{Compliance with Ethical Standards}

The authors declare that they have no conflict of interest. 


\section{References}

1. C. Hollmann, E. Gutheil, Combust. Sci. Tech. 135(1-6), 175 (1998)

2. O. Gicquel, N. Darabiha, D. Thévenin, Proc. Combust. Inst. 28(2), 1901 (2000)

3. J. Van Oijen, F. Lammers, L. de Goey, Combust. Flame 127(3), 2124 (2001)

4. R. Vicquelin, B. Fiorina, S. Payet, N. Darabiha, O. Gicquel, Proc. Combust. Inst. 33(1), 1481 (2011)

5. J. Galpin, A. Naudin, L. Vervisch, C. Angelberger, O. Colin, P. Domingo, Combust. Flame 155(1-2), 247 (2008)

6. B. Fiorina, R. Vicquelin, P. Auzillon, N. Darabiha, O. Gicquel, D. Veynante, Combust. Flame 157(3), 465 (2010)

7. P. Auzillon, E. Riber, L. Gicquel, O. Gicquel, N. Darabiha, D. Veynante, B. Fiorina, C. R. Mécanique 341, 257 (2013)

8. M. Chrigui, J. Gounder, A. Sadiki, A.R. Masri, J. Janicka, Combust. Flame 159(8), 2718 (2012)

9. Y. Baba, R. Kurose, J. Fluid Mech. 612, 45 (2008)

10. B. Franzelli, A. Vié, B. Fiorina, N. Darabiha, in Proceedings of ASME Turbo Expo 2013: Turbine Technical Conference and Exposition, GT2013-94451 (San Antonio, Texas, 2013)

11. A. Vié, B. Franzelli, Y. Gao, T. Lu, H. Wang, M. Ihme, Proc. Combust. Inst. 35(2), 1675 (2015)

12. R. Lecourt, G. Linassier, G. Lavergne, in Proceedings of ASME Turbo Expo 2011: Power for Land, Sea and Air (Vancouver, Canada, 2011)

13. J. Senoner, M. Sanjosé, T. Lederlin, F. Jaegle, M. Garcia, E. Riber, B. Cuenot, L. Gicquel, H. Pitsch, T. Poinsot, C. R. Acad. Sci. 337(6-7), 458 (2009)

14. M. Sanjosé, J. Senoner, F. Jaegle, B. Cuenot, S. Moreau, T. Poinsot, Int. J. Multiphase Flow 37(5), 514 (2011)

15. A. Vié, S. Jay, B. Cuenot, M. Massot, Flow, Turb. and Combustion 90, 545 (2013)

16. B. Franzelli, A. Vié, M. Ihme, Combust. Flame 163, 100 (2016)

17. B. Sirjean, E. Dames, D.A. Sheen, H. Wang, 6th US National Combustion Meeting (2009). Paper 23F1

18. C. Westbrook, F. Dryer, Combust. Sci. Tech. 27(1-2), 31 (1981)

19. W.P. Jones, R.P. Lindstedt, Combust. Flame 73(3), 222 (1988)

20. B. Franzelli, E. Riber, M. Sanjosé, T. Poinsot, Combustion and Flame 157(7), 1364 (2010)

21. B. Franzelli, B. Fiorina, N. Darabiha, Proc. Combust. Inst. 34, 1659 (2013)

22. H.W. Ge, E. Gutheil, Combust. Flame 153(1-2), 173 (2008)

23. K. Luo, H. Pitsch, M. Pai, O. Desjardins, Proc. Combust. Inst. 33(2), 667 (2011)

24. W. Jones, S. Lyra, S. Navarro-Martinez, Combust. Flame 159(4), 1539 (2012)

25. G. Hannebique, P. Sierra, E. Riber, B. Cuenot, Flow, Turb. and Combustion 90(2), 449 (2012)

26. W. Jones, A. Marquis, K. Vogiatzaki, Combustion and Flame 161(1), 222 (2014)

27. S. Ukai, A. Kronenburg, O. Stein, Proc. Combust. Inst. 35(2), 1667 (2015)

28. B. Fiorina, R. Baron, O. Gicquel, D. Thevenin, S. Carpentier, N. Darabiha, Combust. Theory and Modelling 7, 449 (2003)

29. E. Knudsen, H. Pitsch, in Annual Research Briefs (Center for Turbulence Research, NASA Ames/Stanford Univ., 2010), pp. 337-350

30. P.D. Nguyen, L. Vervisch, V. Subramanian, P. Domingo, Combust. Flame 157(1), 43 (2010)

31. P. Schmitt, T.J. Poinsot, B. Schuermans, K. Geigle, J. Fluid Mech. 570, 17 (2007)

32. S. Roux, G. Lartigue, T. Poinsot, U. Meier, C. Bérat, Combust. Flame 141, 40 (2005)

33. O. Colin, F. Ducros, D. Veynante, T. Poinsot, Phys. Fluids 12(7), 1843 (2000)

34. L. Schiller, A. Naumann, V.D.I Zeitung 77, 318 (1935)

35. T. Poinsot, D. Veynante, Theoretical and Numerical Combustion, Third Edition (2012)

36. F. Nicoud, F. Ducros, Flow, Turb. and Combustion 62(3), 183 (1999)

37. F. Charlette, C. Meneveau, D. Veynante, Combust. Flame 131(1-2), 159 (2002)

38. B. Franzelli, E. Riber, B. Cuenot, C. R. Mécanique 341, 247 (2013)

39. D.R. Stull, H. Prophet, Janaf thermochemical tables, 2nd edition. Tech. Rep. NSRDS-NBS 37, US National Bureau of Standards (1971)

40. M. Moreau, O. Simonin, B. Bedat, Flow, Turb. and Combustion 84, 295 (2010)

41. P. Sierra, Modeling the dispersion and evaporation of sprays in aeronautical combustion chambers. Ph.D. thesis, Institut National Polytechnique Toulouse, https://tel.archives-ouvertes.fr/tel-00701105 (2012)

42. G. Hubbard, V. Denny, A. Mills, International Journal of Heat and Mass Transfer 18, 1003 (1975)

43. C.R. Wilke, J. Chem. Phys. 1(191-195) (10950)

44. O. Colin, M. Rudgyard, J. Comput. Phys. 162(2), 338 (2000)

45. T. Poinsot, S. Lele, J. Comput. Phys. 101(1), 104 (1992)

46. N. Darabiha, Combust. Sci. Tech. 86, 163 (1992)

47. N. García-Rosa, Phénomènes d'allumage d'un foyer de turbomachine en conditions de haute altitude. Ph.D. thesis, Institut National Polytechnique Toulouse, http://depozit.isae.fr/theses/2008/2008_Garcia_Rosa_Nicolas.pdf (2008) 
48. M. Sanjosé, Evaluation de la méthode euler-euler pour la simulation numérique aux grandes échelles des chambres à carburant liquide. Ph.D. thesis, Institut National Polytechnique de Toulouse, France (2009)

49. P. Février, O. Simonin, K.D. Squires, J. Fluid Mech. 533, 1 (2005)

50. B. Franzelli, Impact of the chemical description on direct numerical simulation and large eddy simulation of turbulent combustion in industrial aero-engines. Ph.D. thesis, Institut National Polytechnique Toulouse, https://tel.archives-ouvertes.fr/tel-00662813 (2011)

51. A. Attili, F. Bisetti, M.E. Mueller, H. Pitsch, Combustion and Flame 161(7), 1849 (2014)

52. B. Franzelli, E. Riber, B. Cuenot, M. Ihme, in Proceedings of ASME Turbo Expo 2015: Turbine Technical Conference and Exposition, GT2015-43630, (2015)

53. N. Patel, S. Menon, Combust. Flame 153(1-2), 228 (2008)

54. L. Zimmer, T. Providakis, P. Scouflaire, S. Ducruix, in 12th International Conference on Liquid Atomization and Spray Systems (Heidelberg, Germany, 2012) 
Appendix A: Performances of the FPI tabulation method on laminar counterflow spray flames

Franzelli et al. [21] investigated the adequacy of the FPI method to predict laminar counterflow spray flames by comparing in an a priori way the profiles of the tabulated quantities with the detailed variables. The present study completes the 'a priori' analysis presented in [21] by evaluating the performance of the FPI method in a posteriori way on laminar counterflow spray flames (see Fig. 16). The same numerical configuration is considered in the present work. The system of equations describing the laminar spray flow as well as the evaporation model are given in details in [21].

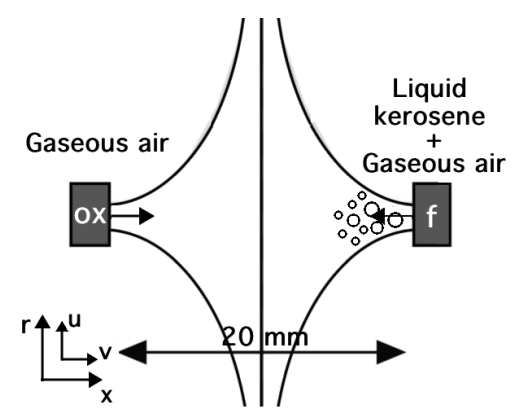

Fig. 16: Schematic of a laminar counterflow spray flame.

Pure fresh air is injected from the left side (superscript $o x$ ) whereas spray fuel and pure air are injected from the right side (superscript $f$ ). Subscripts $g$ and $l$ denote the gas and the liquid phase respectively. The axial gas phase velocities are identical at both injection sides: $v_{g}^{o x}=-v_{g}^{f}$. The axial velocities of the gas and liquid phases at the right injection are equal $v_{g}^{f}=v_{l}^{f}$. Liquid and gas temperatures at injection are equal at both injection sides: $T_{g}^{f}=T_{g}^{o x}=T_{l}=400 \mathrm{~K}$.

1-D flame simulations are performed with the REGATH counterflow code using an EulerEuler approach under the assumption of monodisperse liquid phase [46]. The reader is referred to [21] for more numerical details. Four spray flames, summarized in Table 1, have been investigated for the operating conditions representative of the observed values in the LES (liquid droplet diameter $d_{l}^{f}$, droplet number density $n_{l}^{f}$, injection velocity $v_{l}^{f}$ and liquid volume fraction $\alpha_{l}^{f}$ ).

Table 1: Operating conditions of the different studied cases (value is in bold when the parameter is varied).

\begin{tabular}{|ccccc|}
\hline $\begin{array}{c}\text { Name } \\
\text { case }\end{array}$ & $\begin{array}{c}d_{l}^{f} \\
{[[\mu \mathrm{m}]}\end{array}$ & $\begin{array}{c}\alpha_{l}^{f} \\
{[-]}\end{array}$ & $\begin{array}{c}n_{l}^{f} \\
{\left[1 / \mathrm{m}^{3}\right]}\end{array}$ & $\begin{array}{c}v_{g}^{f} \\
{[\mathrm{~m} / \mathrm{s}]}\end{array}$ \\
\hline $\mathrm{A}$ & 40 & $3.410^{-4}$ & $1.010^{+10}$ & 0.20 \\
$\mathrm{~B}$ & $\mathbf{1 0 0}$ & $3.410^{-4}$ & $\mathbf{0 . 6 4} \mathbf{1 0}^{+\mathbf{9}}$ & 0.20 \\
$\mathrm{C}$ & 40 & $\mathbf{1 . 3 7} \mathbf{1 0}^{-\mathbf{4}}$ & $\mathbf{4 . 0 9} \mathbf{1 0}^{+\mathbf{9}}$ & 0.20 \\
$\mathrm{D}$ & 40 & $3.410^{-4}$ & $1.010^{+10}$ & $\mathbf{1 . 0 0}$ \\
\hline
\end{tabular}


The look-up table is built from a collection of 100 adiabatic unstrained gaseous premixed flames for 100 and 500 different values of $Y_{z}$ and $Y_{c}$, respectively, at $T^{f}=400 \mathrm{~K}$ and ambient pressure using the reference detailed chemical scheme [21]. Results obtained with the tabulated approach (lines) are compared to the multi-species description (symbols) in Fig. 17. In the left side of Fig. 17, the flame structure is represented by the mixture fraction and the progress variable profiles, together with the information of the liquid volume fraction $\alpha_{l}$. Temperature and $C O$ mass fraction profiles are provided on the right side of Fig. 17.

The chemical structure of the counterflow spray flame is shown in Fig. 17(a) for case A in Table 1. Near the flame front, the gas temperature increases due to the thermal conductivity. Consequently, the evaporation source term drastically increases and the liquid volume fraction completely evaporates. The high temperature region $(-5 \mathrm{~mm}<x<3 \mathrm{~mm})$ is characterized by the presence of intermediate species, such as $\mathrm{CO}$, and products. A good agreement is observed between the detailed multi-species solution and the tabulated chemistry technique. The FPI method correctly localizes the flame front. However, as only a single flame archetype is used to generate the look-up table which does not account for strain rate and non-premixed effect, the temperature is slightly underestimated and the $\mathrm{CO}$ mass fraction is overestimated in rich regions [21].

In case $\mathrm{B}$, the droplet diameter is increased keeping constant the liquid volume fraction at injection by decreasing the droplet number density (Fig. 17(b)). Since the droplets are bigger, evaporation is initially slower and fuel remains mainly in liquid phase before reaching the flame front, resulting in higher gradient of the mixture fraction in the high temperature region, where the liquid evaporates rapidly. Globally, the same agreement between the detailed description and the tabulated method discussed for case A is observed here.

The impact of the liquid volume fraction $\alpha_{l}^{f}$ on the flame structure and the performances of the tabulation method is investigated in case $\mathrm{C}$ (Table 1). The liquid volume fraction $\alpha_{l}^{f}$ is decreased keeping constant the droplet diameter, which means that the overall equivalence ratio is reduced. Evaporation is mainly localized before the flame front (Fig. 17(c)). As the combustion mainly occurs under premixed conditions, the FPI tabulation procedure is well adapted, leading to a good prediction of the temperature and $\mathrm{CO}$ mass fraction.

Finally, the injection velocity of both liquid and gas phases have been increased in case D (Table 1), keeping constant all other boundary conditions (Fig. 17(d)). The reaction zone is correctly located but the maximum value of the mixture fraction is overestimated. The temperature is correctly described in the near-injection zone as well as the liquid volume fraction. On the contrary, the $\mathrm{CO}$ mass fraction is overestimated by the tabulated methods. Globally, the FPI approach correctly reproduces the flame structure (i.e. $Y_{c}$ and $Y_{z}$ spatial evolution) for counterflow spray flames. However, a more sophisticated approach based on the tabulation of multiple maniforld is necessary to obtain a good prediction of $\mathrm{CO}$ in laminar spray flames in order to account for the effect of strain rate and of the mixture fraction inhomogeneities on pollutant predictions. 

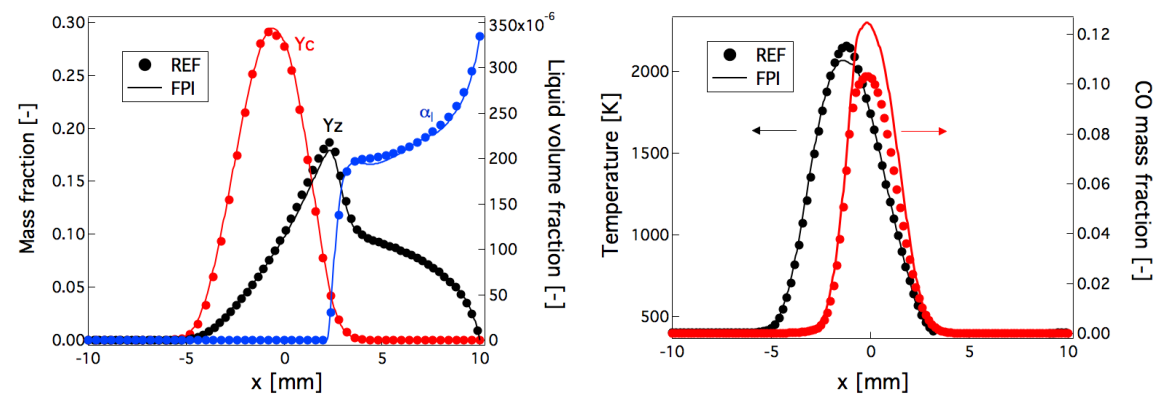

(a) Case $\mathrm{A}$
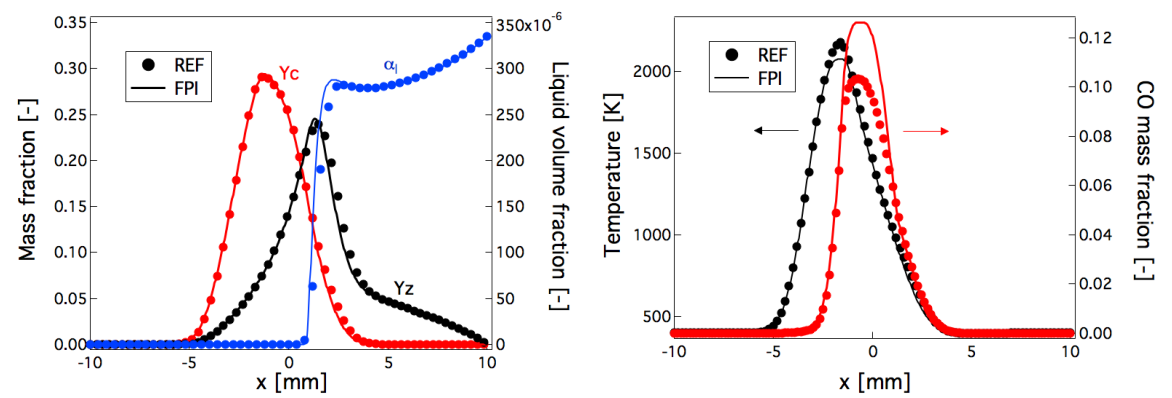

(b) Case B
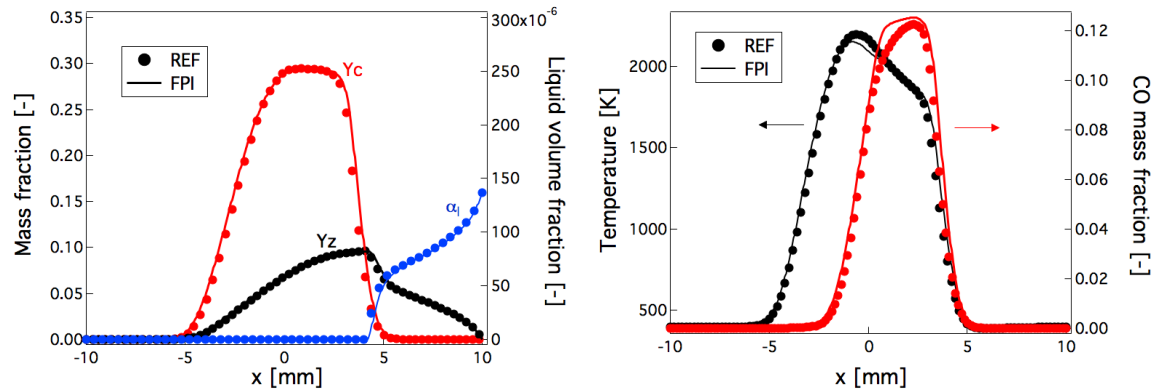

(c) Case $\mathrm{C}$
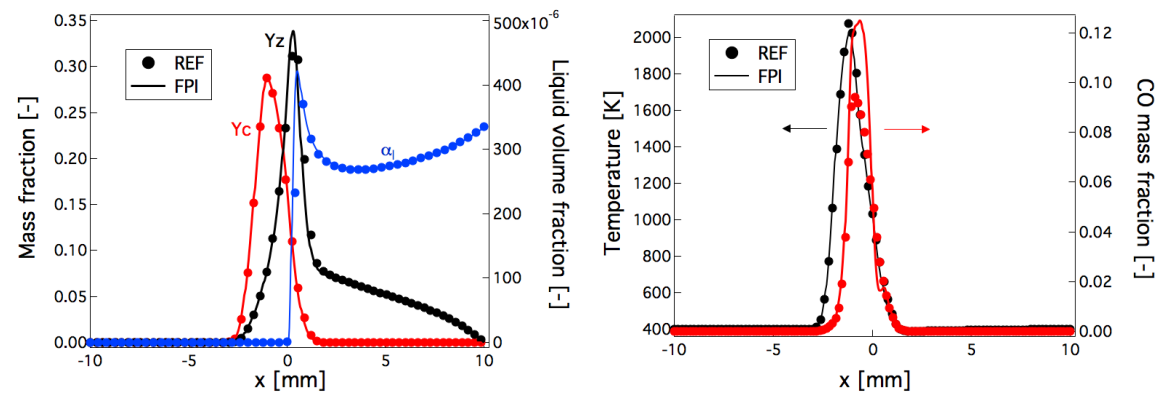

(d) Case D

Fig. 17: Counterflow laminar spray flames of Table 1 (A-D from top to bottom). Comparison between the reference detailed model (symbols) and the tabulated description (line): axial profiles of mixture fraction, progress variable and liquid volume fraction (left), temperature and $\mathrm{CO}$ mass fraction (right). 